\title{
Systematic Review of Chinese Herbal Medicines for Preventing in-Stent Coronary Restenosis after Percutaneous Coronary Intervention
}

\author{
Guo-Hua Zheng, ${ }^{1}$ Jian-Ping Liu, ${ }^{2}$ Nissi S. Wang, ${ }^{3}$ Hai-Ying Chen, ${ }^{1}$ and Jian-Feng Chu ${ }^{1}$ \\ ${ }^{1}$ The Centre of Evidence Based Medicine, Academy of Integrative Medicine, Fujian University of Traditional Chinese Medicine, \\ NO. 1 Huatuo Road, Shangjie University Town, Fuzhou 350108, China \\ ${ }^{2}$ The Centre for Evidence-Based Chinese Medicine, Beijing University of Chinese Medicine, Beijing 100029, China \\ ${ }^{3}$ Pamir Communications, Daly City, CA 94015, USA
}

Correspondence should be addressed to Jian-Ping Liu, jianping_l@hotmail.com

Received 18 August 2011; Accepted 6 December 2011

Academic Editor: Myeong Soo Lee

Copyright (c) 2012 Guo-Hua Zheng et al. This is an open access article distributed under the Creative Commons Attribution License, which permits unrestricted use, distribution, and reproduction in any medium, provided the original work is properly cited.

Percutaneous coronary intervention (PCI) with stent placement is a standard treatment for coronary artery disease (CAD). Instent restenosis after PCI remains a challenging clinical problem. In China, Chinese herbal medicines (CHMs) are widely used for preventing restenosis. This paper systematically reviewed the literature on the effectiveness and safety of CHMs in preventing restenosis after PCI in patients with CAD. Electronic databases were searched for randomized controlled trials that compared CHMs plus RWM with the same RWM plus placebo in preventing restenosis after PCI. A total of 52 trials (4905 patients) on 34 CHMs met the inclusion criteria and were analyzed. Ten trials had low risk of bias. Methodological quality of included trials was generally poor. Meta-analysis showed that at the end of at least 3 months' followup, CHMs plus RWM could significantly reduce restenosis rate, cardiac mortality, recurrence rate of angina, acute myocardial infarction, numbers of repeat PCI, and numbers of coronary artery bypass graft. Reported adverse events included gastrointestinal upset, granulocytopenia, and increased alanine transaminase (ALT). CHMs may help prevent restenosis, thus reducing cardiac mortality after PCI. Caution should be exercised in drawing a definitive conclusion due to the poor methodological quality of the trials reviewed.

\section{Introduction}

Coronary artery disease (CAD) is the single leading cause of death and disability in the world [1-3]. Percutaneous coronary intervention (PCI) with stent placement is the standard nonsurgical treatment for $\mathrm{CAD}$, effective in relieving the symptoms of coronary ischemia [4]. But the main limitation of coronary stenting, in particular with bare-metal stents, is in-stent restenosis (ISR) [5]. This is the formation of scar tissue over the stent, which can cause the opened artery to narrow again. The risks of ISR include symptoms of coronary ischemia, often warranting repeat revascularization [6]. With the development and the universal application of the drugeluting stent (DES), restenosis has been reduced from $10 \%$ to $50 \%$ for bare-metal stents [7] to $<10 \%$ for DESs [8]. Despite this improvement, a major drawback of DESs has come to light involving late (after 30 days) and very late (after 1 year) stent thrombosis $[9,10]$. In addition, studies have shown that DESs do not reduce late cardiac-related death and the incidence of myocardial infarction (MI) [11-14]. Antithrombotic therapy after PCI, consisting of lifelong aspirin and clopidogrel, is effective in reducing cardiac-related death, MI, and stroke [15]. But dual antiplatelet therapy also has limitations, as stopping prematurely significantly increases the risk of stent thrombosis, MI, and death [16]. Thus, treatment of ISR remains a challenging clinical issue.

In China, Chinese herbal medicines CHMs have a long history of integration with routine Western medical (conventional) interventions. Advancements in such interventions have spurred concomitant application of CHMs in attempts to enhance outcomes. In the past decade, CHMs have been tested in clinical trials as an adjunct therapy for preventing 
ISR after PCI. CHMs appear to ameliorate ISR after PCI when used alone or combined with routine western medicine (RWM) [16, 17]. Pharmacologic studies have found that some (CHMs) can be administered to dilate coronary vessels, improve circulation, and remove blood stasis (Chinese medicine concept of blood circulation disturbance, resulting in slowing of blood flow, thrombosis, retained blood). Additionally, these CHMs possess antiinflammatory, immune response inhibition, anti-platelet aggregation, and antiperoxidative properties, as well as functions that inhibit proliferation and migration of vascular smooth muscle cells [18-21]. Reviews on the efficacy of CHMs in preventing restenosis after PCI have been published in Chinese [22-24]. But the evidence supporting or disproving the benefits of $\mathrm{CHMs}$ is not robust because of methodological deficiencies of those reviews. Our study presents a more vigorous attempt to examine the existing studies to draw more useful conclusions about the safety and efficacy of CHMs in preventing restenosis post-PCI.

\section{Methods}

2.1. Inclusion Criteria. Only randomized controlled trials (RCTs) were included regardless of being published or unpublished. We focused on trials with participants diagnosed with major angiographic criteria-documented [25] coronary artery disease who were eligible for PCI regardless of gender, age, and ethnic origin.

The treatment group in the trials was treated with any $\mathrm{CHM}$, or CHM plus RWM with at least 1 month of therapy regardless of dosage. The control group was treated with the same RWM based on the Chinese Society of Cardiology guidelines for percutaneous transluminal coronary intervention [25], or the same RWM plus placebo. "Chinese herbal medicines" include extracts from mixtures of herbs, single herbs, Chinese prepared medicines, or a compound of herbs that is prescribed by a Chinese medicine practitioner [26].

Primary outcome measures were restenosis, cardiac death, and adverse events occurring in at least 3 months of followup. Secondary outcome measures included recurrent angina, acute myocardial infarction (AMI), revascularization, repeat PCI, coronary artery bypass graft (CABG), minimal luminal diameter (MLD), late loss of lumen (LLL), net gain in lumen diameter (NG), and lesion area net gain (LANG) after PCI, and quality of life during at least 1 month of followup.

\subsection{Study Identification and Assessment in Included Studies.} Two authors (G.-H. Zheng, H.-Y. Chen) independently identified studies through searches of the Cochrane Library (Issue 4, 2010), PubMed (January1966 to December 2010), Embase (January 1980 to December 2010), the China Biological Medicine Database (CBM, January 1980 to December 2010), Chinese Scientific Journal Database (VIP, January1989 to December 2010), China National Knowledge Information database (CNKI, January 1994 to December 2010) and Chinese Medical Citation Index (CMCI, January 1999 to December 2010) with free terms related to heart disease and CHM (e.g., "coronary heart disease" OR "coronary artery disease" OR "cardiovascular disease" OR "percutaneous coronary intervention" OR "stent" OR "stenosis" OR "restenosis" OR "CHD" OR "CVD" OR "CAD" OR "MI" "Chinese" OR "herbal" OR for English databases. The Chinese counterpart terms were used for Chinese databases. The reference list of each relevant article was searched for further studies. Unpublished literature was searched using Chinese Master's Theses Full-text Database (CMFD), China Doctor Dissertation Full-text Database (CDFD) and China Proceedings of Conference Full-text Database (CPCD).

Risk of bias in included studies was assessed using The Cochrane Collaboration's tool for assessing risk of bias. Six criteria were applied: adequate sequence generation, concealment of allocation, blinded of primary outcomes, adequately addressed incomplete outcome data, free from selective reporting, and free of other risk of bias [27]. In addition, we assessed the baseline characteristics between the comparison groups.

2.3. Data Extraction. Two authors (G.-H. Zheng, J.-F. Chu) independently selected those trials that met the inclusion criteria and extracted details on randomization, allocation concealment, blinding, intent to treat analysis, numbers lost to followup, patient demographics, methods, interventions, outcomes, and results. Missing data were obtained from the original authors when possible.

2.4. Data Analysis. Heterogeneity across studies was tested using a standard $\chi^{2}$ test [28] and Higgins $I^{2}$ [29]. When heterogeneity was not significant $(P \geq 0.1)$, the results were pooled using a fixed effect model and the MantelHaenszel test. Otherwise, a random effect model and the Dersimonian and Laird method were applied [30]. The results were reported as risk ratio (RR) with corresponding 95\% confidence interval (CI) for dichotomous data. If continuous data were available, weighted mean difference or standardized mean difference was calculated [31]. All data were analyzed using the statistical software RevMan 5.0.1 (Oxford, England) of The Cochrane Collaboration, and all $P$ values were two sided.

\section{Results}

3.1. Study Identification. Eligible literature was screened and identified (Figure 1). A total of 806 records were retrieved. Of these, full-text evaluation was conducted on 154 studies. This was followed by elimination of 102 studies: irrelevant to CHM ( $n=61)$; irrelevant to the primary or secondary outcomes $(n=10)$; control group combined with another CHM $(n=23)$; duplicate publication $(n=6)$ [32-37]; primary outcomes $<3$ months' followup $(n=1)$ [38]; CHM treatment $<1$ month treatment $(n=1)$ [39]. Finally, 52 RCTs with a total of 4905 patients in treatment and control groups, fulfilled the inclusion criteria [40-91]. All studies were conducted in China from 1979 to 2010.

3.2. Characteristics of Included RCTs. Table 1 summarizes the characteristics of included studies. Average age of patients in the included studies ranged from 51.2 to 72 years. Each 


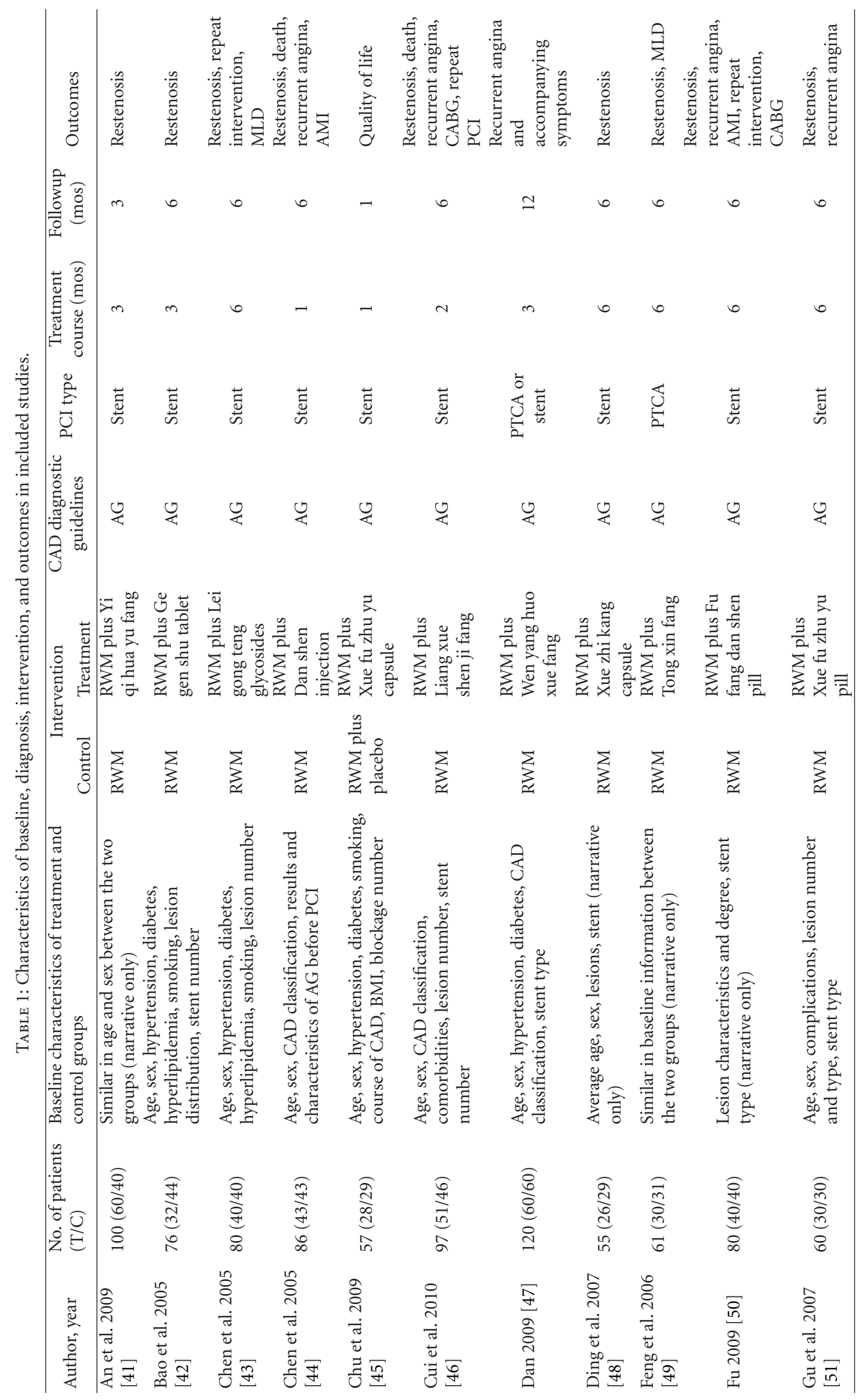




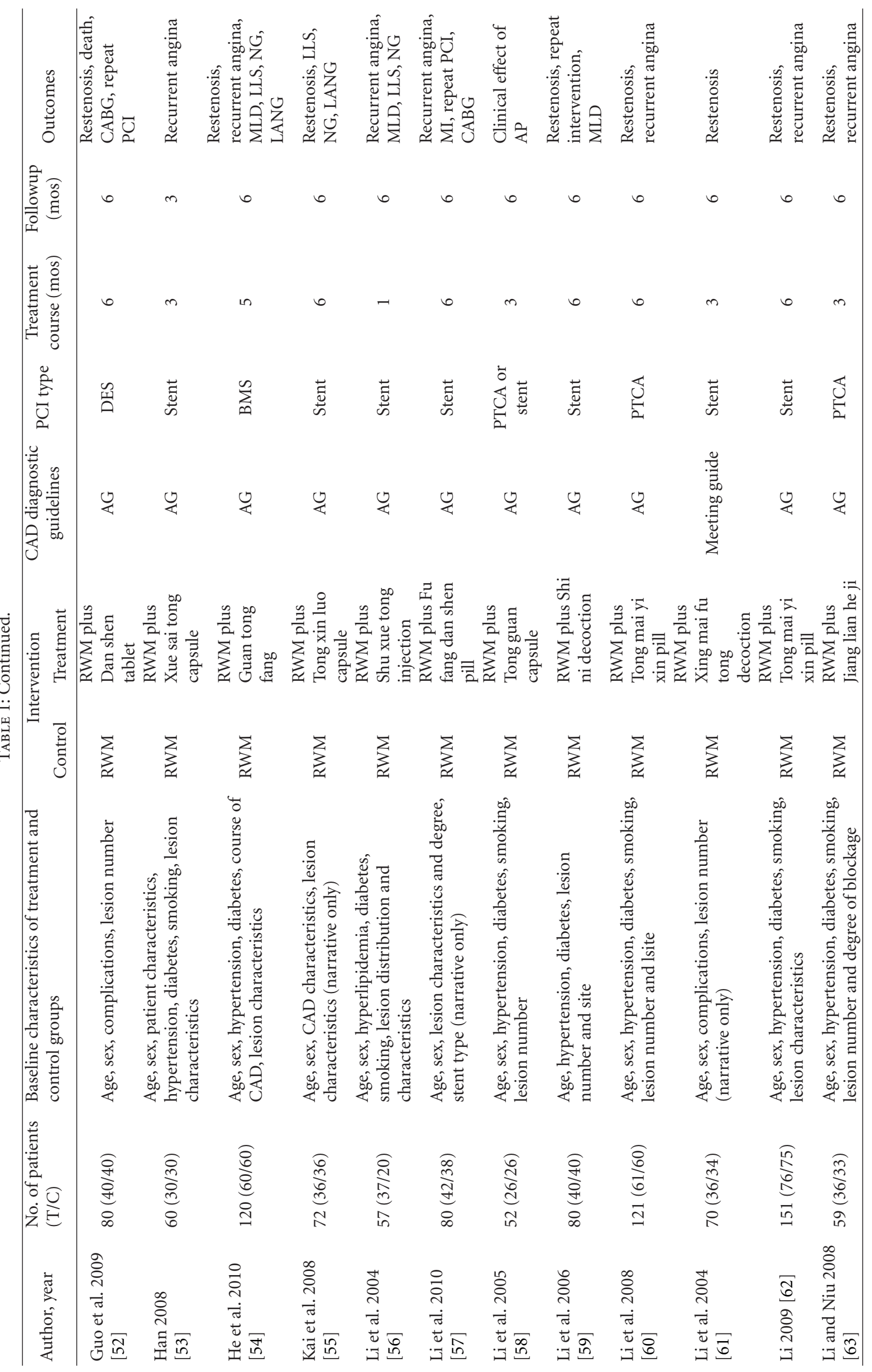




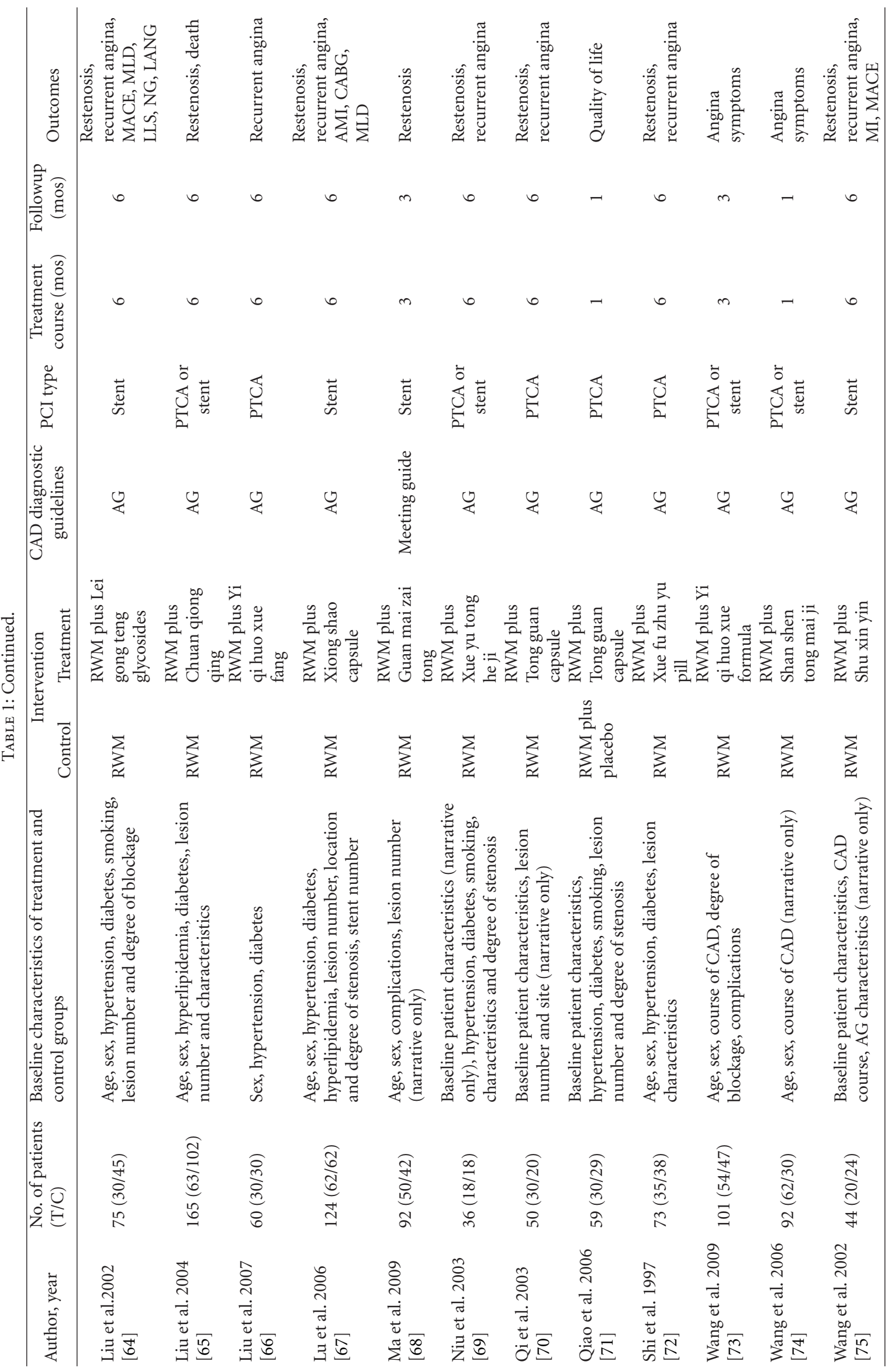




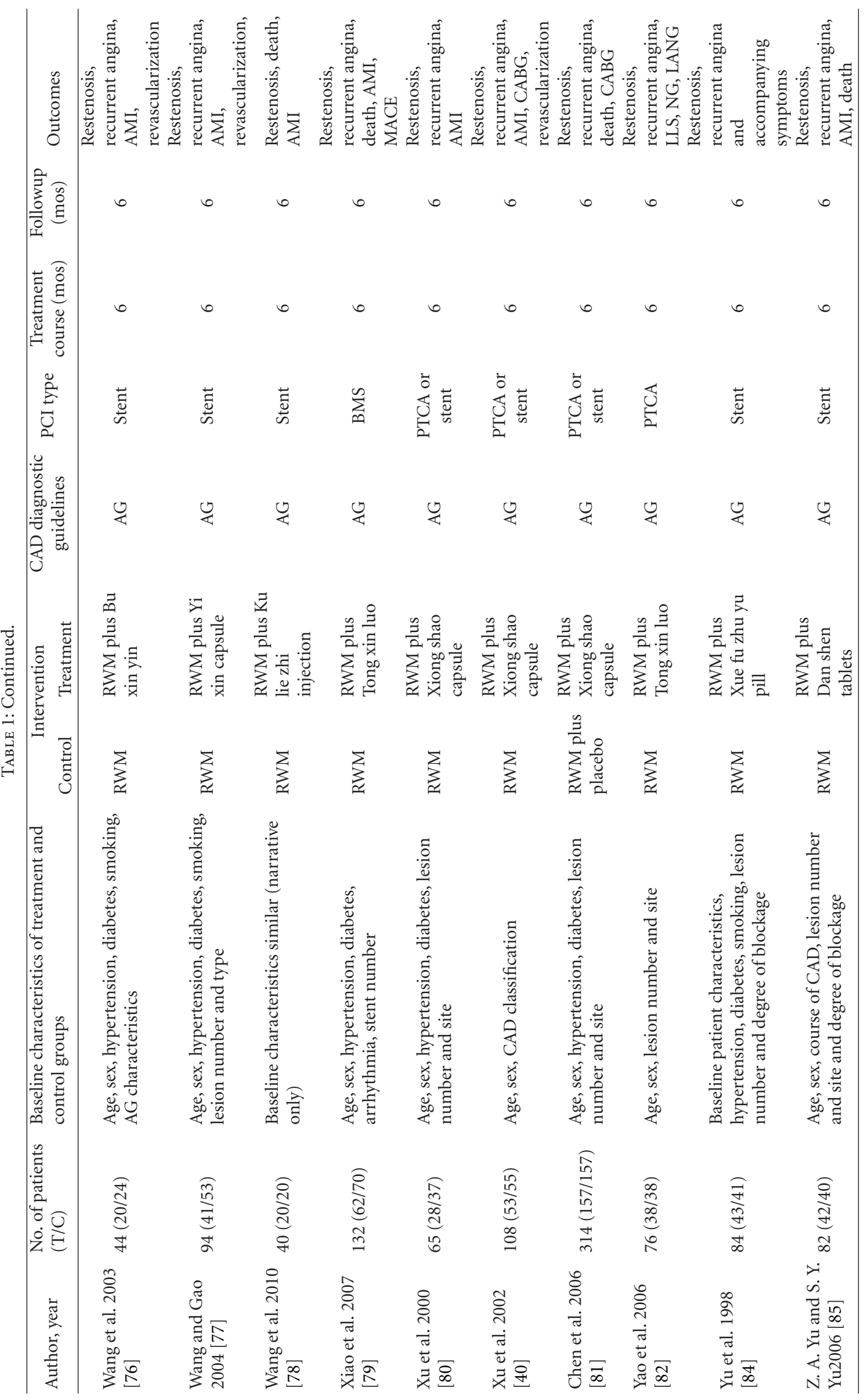




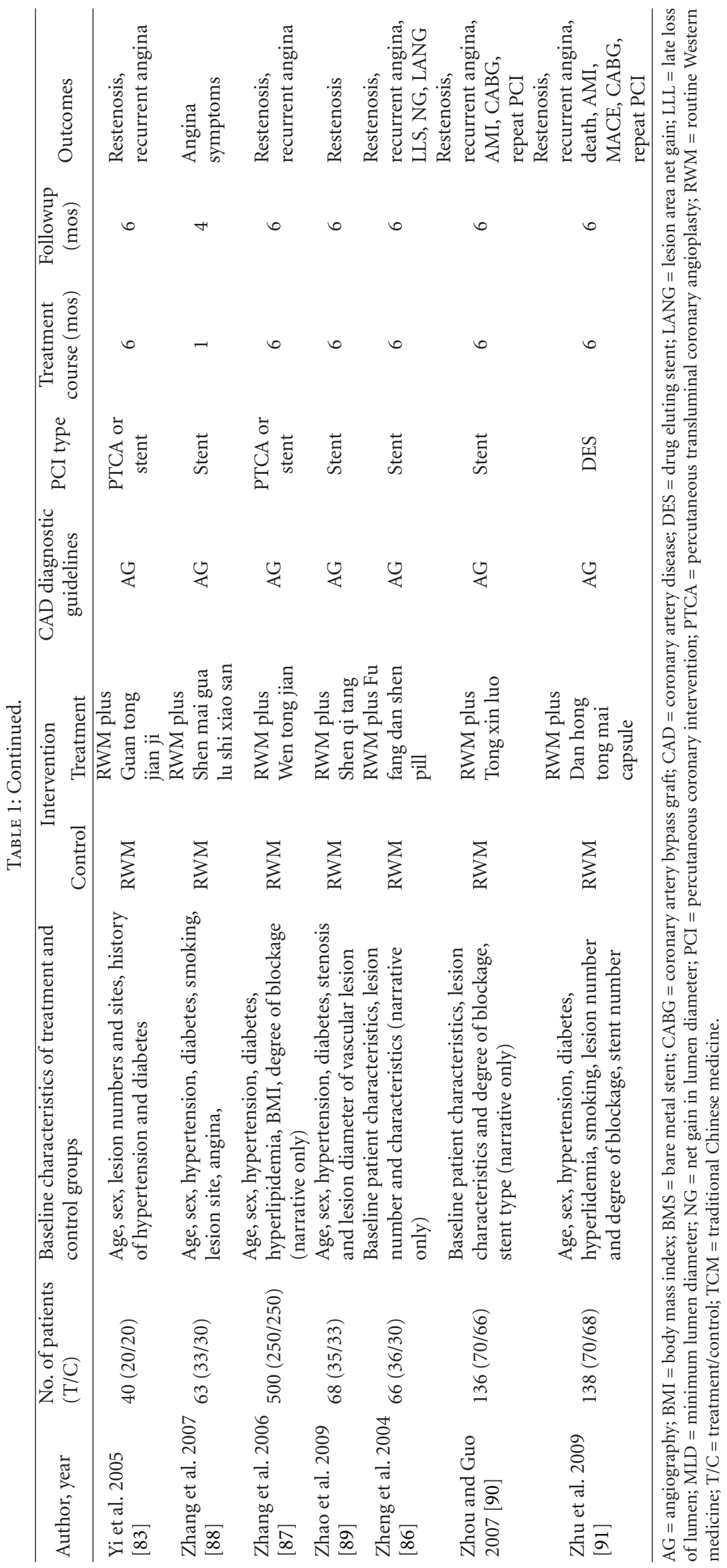




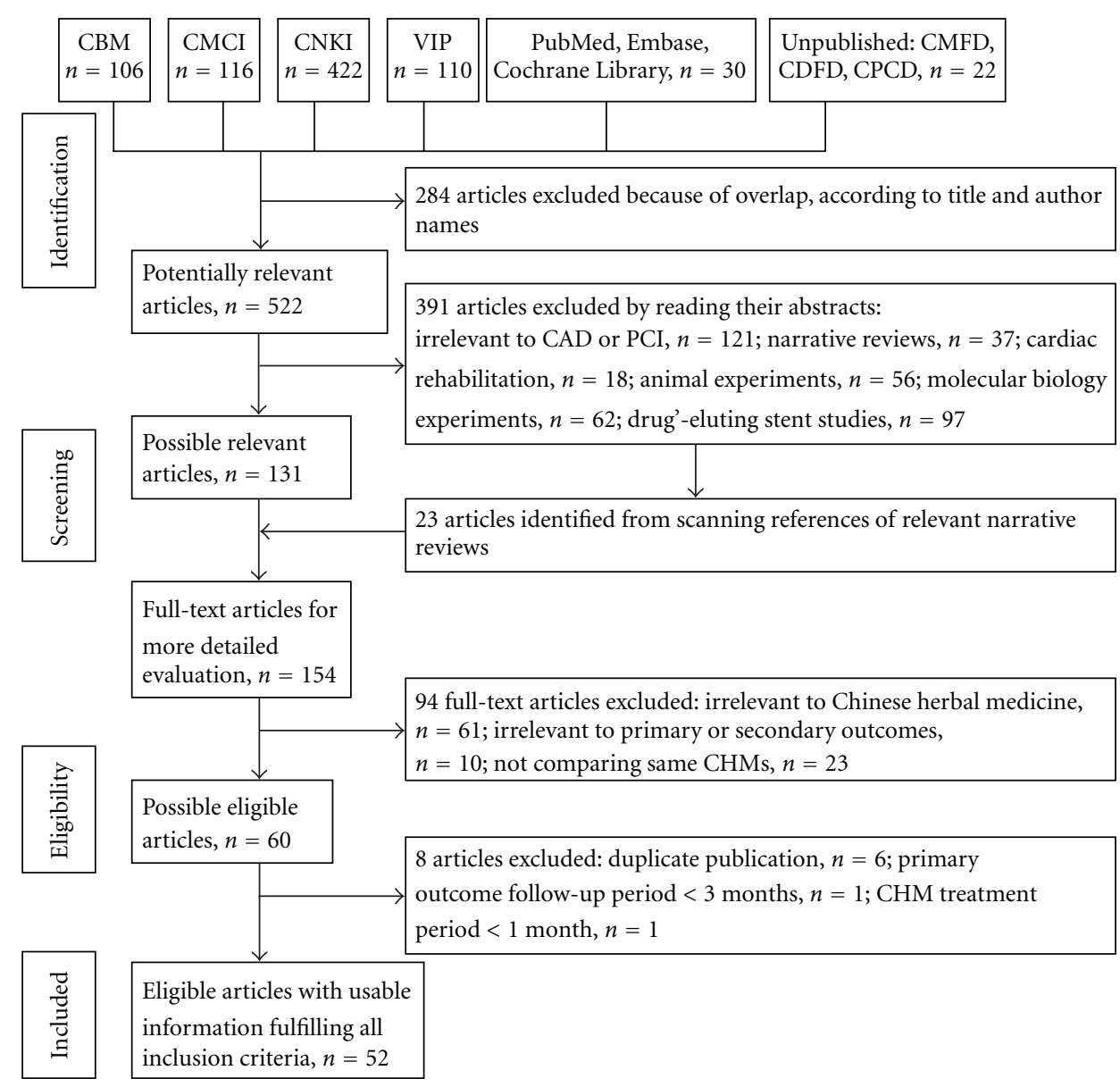

FIgURe 1: Literature search and selection. Abbreviations: CBM: China Biological Medicine Database; CDFD: China Doctor Dissertation Full-Text Database; CMCI: Chinese Medical Citation Index; CMFD: Chinese Master's Theses Full-Text Databases; CNKI: China National Knowledge Infrastructure; CPCD: China Proceedings of Conference Full-text Database; VIP: VIP Database.

trial had more males than females. The diagnostic criteria of CAD were mainly based on coronary angiography criteria. All patients successfully underwent PCI.

Four trials $[45,67,71,81]$ were randomized double blind, and placebo controlled comparing RWM plus CHM versus the same RWM plus placebo. The remaining trials were designed comparing CHM plus RWM versus the same RWM alone. The dosage and types of RWM were prescribed according to Chinese Society of Cardiology guideline recommendations [25]. In the trials, 34 kinds of CHMs were used, and the period of treatment with a CHM was at least 1 month. Followup after PCI ranged from 3 to 12 months, with 6 months in the majority of studies.

Restenosis was assessed using angiography in 40 trials. Of these, 10 studies reported angiography assessments at the end of at least 3 months after PCI. Adverse events caused by CHMs were reported in 21 studies, but none of the studies underwent statistical analysis. Recurrent angina was reported in 33 studies. Major cardiac events were reported in 16 trials, and 10 trials reported cardiac death after followup of at least 6 months. Only 2 studies assessed quality of life using the Short-Form 36 (SF-36) Health Survey and Seattle Angina Questionnaire (SAQ) at the end of 1 month after PCI.

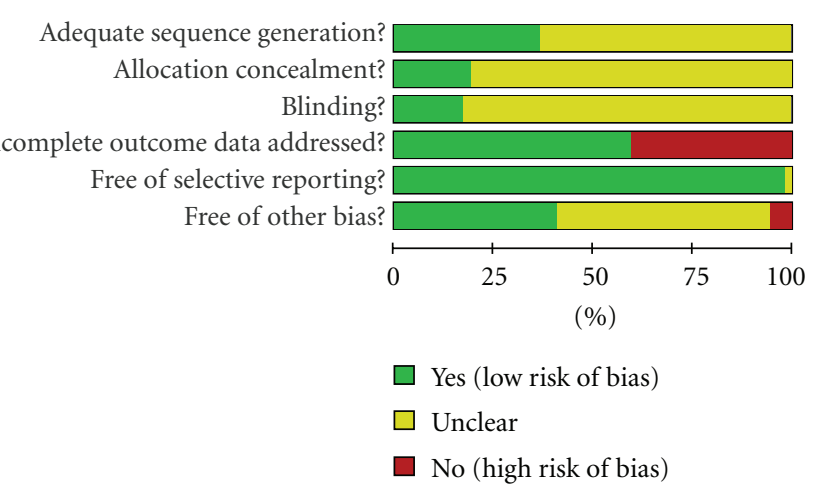

FIGURE 2: Risk of bias graph depicting proportions of studies with each judgment.

3.3. Methodological Quality of Included RCTs. Risk of bias in the studies is shown in Figure 2. Of the 52 studies, 19 studies reported randomization using random number tables or computer random number generator such as SAS. The remaining 33 studies reported "randomly allocating" participants, but the method of randomization was not 
TABLE 2: Risk ratios of Chinese herbal medicines administered to prevent restenosis after PCI.

\begin{tabular}{|c|c|c|c|c|}
\hline Chinese herbal medicine & Number of studies & Number of patients & Risk ratio (RR) & $95 \% \mathrm{CI}$ of $\mathrm{RR}$ \\
\hline Bu xin yin plus RWM versus RWM & $1[76]$ & 44 & 0.69 & $0.23 \sim 2.01$ \\
\hline Chuan qiong qing plus RWM versus RWM & $1[65]$ & 165 & 0.51 & $0.19 \sim 1.31$ \\
\hline Dan hong tong mai capsule plus RWM versus RWM & $1[91]$ & 103 & 0.35 & $0.10 \sim 1.23$ \\
\hline Dan shen plus RWM versus RWM & $5[44,50,52,85,86]$ & 388 & 0.27 & $0.14 \sim 0.52$ \\
\hline Ge gen shu plus RWM versus RWM & $1[42]$ & 76 & 0.23 & $0.06 \sim 0.95$ \\
\hline Guan mai zhai tong decoction plus RWM versus RWM & $1[68]$ & 92 & 0.32 & $0.09 \sim 1.11$ \\
\hline Guan tong formula plus RWM versus RWM & $1[54]$ & 120 & 0.43 & $0.18 \sim 1.04$ \\
\hline Jia wei xue fu zhu yu particle plus RWM versus RWM & $1[51]$ & 40 & 0.75 & $0.27 \sim 2.07$ \\
\hline Jiang lian he ji plus RWM versus RWM & $1[63]$ & 26 & 0.57 & $0.11 \sim 2.87$ \\
\hline Ku lie zhi injection plus RWM versus RWM & $1[78]$ & 40 & 1.00 & $0.07 \sim 14.9$ \\
\hline Lei gong teng plus RWM versus RWM & $2[43,64]$ & 133 & 0.75 & $0.40 \sim 1.40$ \\
\hline Liang xue shen ji formula plus RWM versus RWM & $1[46]$ & 97 & 0.45 & $0.12 \sim 1.70$ \\
\hline Shen qi decoction plus RWM versus RWM & $1[89]$ & 68 & 0.47 & $0.09 \sim 2.40$ \\
\hline Shu xin yin plus RWM versus RWM & $1[75]$ & 44 & 0.69 & $0.23 \sim 2.01$ \\
\hline Si ni tang plus RWM versus RWM & $1[59]$ & 63 & 0.55 & $0.14 \sim 2.09$ \\
\hline Tong guan capsule plus RWM versus RWM & $1[70]$ & 25 & 0.67 & $0.26 \sim 1.72$ \\
\hline Tong mai yu xin plus RWM versus RWM & $2[60,62]$ & 272 & 0.39 & $0.21 \sim 0.70$ \\
\hline Tong xin fang plus RWM versus RWM & $1[49]$ & 61 & 1.03 & $0.23 \sim 4.72$ \\
\hline Tong xin luo plus RWM versus RWM & $4[39,79,82,90]$ & 328 & 0.34 & $0.20 \sim 0.59$ \\
\hline Wen tong jian plus RWM versus RWM & $1[87]$ & 467 & 0.03 & $0.00 \sim 0.49$ \\
\hline Xue fu zhu yu pin plus RWM versus RWM & $2[72,84]$ & 157 & 0.61 & $0.32 \sim 1.16$ \\
\hline Xue yu tong he ji plus RWM versus RWM & $1[69]$ & 36 & 1.0 & $0.16 \sim 6.35$ \\
\hline Xue zhi kang plus RWM versus RWM & $1[48]$ & 55 & 0.30 & $0.09 \sim 0.99$ \\
\hline Xing mai fu tong decoction plus RWM versus RWM & $1[61]$ & 70 & 0.71 & $0.17 \sim 2.94$ \\
\hline Xiong shao capsule plus RWM versus RWM & $2[40,80]$ & 173 & 0.43 & $0.23 \sim 0.81$ \\
\hline Yi qi hua yu formula plus RWM versus RWM & $1[41]$ & 100 & 0.13 & $0.02 \sim 1.10$ \\
\hline Yi xin capsule plus RWM versus RWM & $1[77]$ & 94 & 0.50 & $0.27 \sim 0.91$ \\
\hline Self-prepared guantong decoction plus RWM versus RWM & $1[83]$ & 30 & 0.40 & $0.16 \sim 1.00$ \\
\hline Overall (CHMs plus RWM versus RWM) & 38 & 3367 & 0.43 & $0.36 \sim 0.51$ \\
\hline Xiong shao capsule plus RWM versus RWM plus placebo & $2[67,81]$ & 438 & 0.46 & $0.27 \sim 0.76$ \\
\hline Overall (CHMs plus RWM versus RWM plus placebo) & 2 & 438 & 0.46 & $0.27 \sim 0.76$ \\
\hline
\end{tabular}

$\mathrm{CHM}=$ Chinese herbal medicine; $\mathrm{CI}=$ confidence interval; $\mathrm{RWM}=$ routine Western medicine.

described. Allocation concealment in 10 of the 52 studies was by sealed, opaque envelopes. In 9 trials, participants and/or outcome assessors were blinded. In most studies, data collection was clearly described and reported, so we judged them as free of selective reporting outcomes. We graded 28 of the 52 studies as "unclear" in terms of free of other bias because there was no evidence of statistical testing of baseline characteristics between comparison groups or the outcome data were incomplete. As a whole, 10 studies [44$46,53,67,71,75,76,81,85]$ had low risk of bias with high methodological quality. Most studies were found to be high risk of bias with low methodological quality.

\subsection{Measures of Effect}

3.4.1. Restenosis. In 40 studies on 29 Chinese herbal medicines (CHMs) comprised of 3805 patients, restenosis was assessed using coronary angiography after PCI with at least 3 months' followup (Table 2). Compared to RWM alone, the rate of restenosis was clearly low in patients administered the same RWM plus CHMs. Of 29 CHMs, 9 CHMs (including Xiong shao capsule, Dan shen, Ge gen shu, Tong mai yu xin pill, Tong xin luo, Wen tong jian, Xue zhi kang, Yi xin capsule, and self-prepared guan tong decoction) showed significant ability in reducing restenosis. In particular, two studies $[67,81]$ on Xiongshao capsule (438 patients) appeared to have reliable results because their designs were randomized, double blinded, and placebo controlled. The overall risk ratio (RR) of restenosis was 0.46 with $95 \% \mathrm{CI}=0.27$ to 0.76 compared to placebo.

3.4.2. Cardiac Mortality. In 10 studies on $9 \mathrm{CHMs}$, comprised of 628 patients in the treatment group and 667 patients in the control group, cardiac death was included as a measure of effect at the end of 6 months of follow-up after PCI. A pooled analysis of CHM plus RWM versus the same RWM found a statistically significant decrease in risk of cardiac death associated with CHM, though we did not 
TABLe 3: Risk ratios of Chinese herbal medicines administered to prevent cardiac mortality after PCI.

\begin{tabular}{|c|c|c|c|c|}
\hline Chinese herbal medicine & Number of studies & Number of patients & Risk ratio (RR) & $95 \% \mathrm{CI}$ of $\mathrm{RR}$ \\
\hline $\mathrm{Bu}$ xin yin plus RWM versus $\mathrm{RWM}$ & $1[76]$ & 44 & 0.4 & $0.02 \sim 9.24$ \\
\hline Chuan qiong qing plus RWM versus RWM & $1[65]$ & 165 & 0.23 & $0.01 \sim 4.38$ \\
\hline Dan hong tong mai capsule plus RWM versus RWM & $1[91]$ & 138 & 2.92 & $0.12 \sim 70.35$ \\
\hline Dan shen plus RWM versus RWM & $2[44,85]$ & 168 & 0.33 & $0.03 \sim 3.07$ \\
\hline Ku lie zhi injection plus RWM versus RWM & $1[78]$ & 40 & 0.33 & $0.01 \sim 7.72$ \\
\hline Liang xue shen ji formula plus RWM versus RWM & $1[46]$ & 97 & 0.15 & $0.02 \sim 1.2$ \\
\hline Shu xin yin plus RWM versus RWM & $1[75]$ & 44 & 0.40 & $0.02 \sim 9.24$ \\
\hline Tong xin luo plus RWM versus RWM & $1[79]$ & 132 & 0.38 & $0.02 \sim 9.06$ \\
\hline Wen tong jian plus RWM versus RWM & $1[87]$ & 467 & 0.07 & $0.00 \sim 1.32$ \\
\hline Overall (CHMs plus RWM versus RWM) & 10 & 1295 & 0.27 & $0.11 \sim 0.63$ \\
\hline
\end{tabular}

$\mathrm{CHM}=$ Chinese herbal medicine; $\mathrm{CI}=$ confidence interval; $\mathrm{RWM}=$ routine Western medicine.

find a significant difference upon subgroup analysis based on different CHMs (Table 3).

3.4.3. Adverse Effects. Adverse effects due to CHMs were not mentioned in 30 studies. In 14 studies, the authors reported that there were no noteworthy adverse events. The remaining 8 studies on 7 CHMs reported adverse events, including gastrointestinal upset, granulocytopenia, elevated alanine transaminase (ALT), aphthous stomatitis, skin pruritus, papular urticaria. Most adverse events were not severe and disappeared without special treatment. With the CHM Lei gong teng, adverse events in the treatment group were higher than those in the control group $(\mathrm{RR}=37.02$, $95 \% \mathrm{CI}=2.29$ to 597.88 ). With CHM Si ni tang, adverse events in the treatment group were lower than that of the control group $(\mathrm{RR}=0.38,95 \% \mathrm{CI}=0.21$ to 0.69$)$. There was no statistically significant difference between the two groups in the remaining $5 \mathrm{CHMs}$. Pooled analysis could not be done in 8 studies on 7 CHMs because of heterogeneity among the studies.

3.4.4. Recurrent Angina. Recurrent angina 6 months postPCI was reported as a measure of effect in 33 studies comprised of 3375 patients (Table 4). The rate of recurrent angina in the RWM plus CHM group was lower than that of the same RWM alone group for the CHMs Dan hong tong mai capsule, Dan shen, Guan tong formula, Lei gong teng, Tong mai yi xin pill, Tong xin luo, Wen tong jian, Xiong shao capsule, Xue fu zhu yu pill, Xue zhi kang, Wen yang huo xue formula. A statistically significant difference was not observed for each of the remaining CHMs. Pooled results of these trials showed recurrent angina was significantly decreased. For Xiong shao capsule, risk of recurrent angina was clearly decreased in the CHM plus RWM group than in the same RWM plus placebo control group.

\subsubsection{Major Adverse Cardiac Effects}

Acute Myocardial Infarction (AMI). In 15 studies on 9 CHMs, involving 1551 patients, acute myocardial infarction was reported as a measure of effect 6 months after PCI (Table 5). No statistically significant difference was found in each study, but meta-analysis did show decreasing incidence of $\mathrm{AMI}$ in the CHM plus RWM group $(\mathrm{RR}=0.22,95 \% \mathrm{CI}=$ 0.1 to 0.49 ).

Revascularization. Revascularization after the index PCI was reported as a measure of effect in 6 studies on 4 CHMs (Shu xin yin, Bu xin yin, Yi xin capsule, and Xiong shao capsule) comprised of 716 patients (Table 5). Numbers of revascularization in the CHM plus RWM group was not significantly lower than that in RWM alone group $(\mathrm{RR}=$ $0.64,95 \% \mathrm{CI}=0.38$ to 1.08 ). However, compared to RWM plus placebo, administering CHM Xiong shao capsule plus the same RWM (2 studies with 426 patients) clearly reduced the numbers of revascularization $(\mathrm{RR}=0.48,95 \% \mathrm{CI}=0.30$ to $0.78)$.

Repeat PCI. Repeat PCI was reported as a measure of effect in 9 studies, which involved $8 \mathrm{CHMs}$ with 807 patients (Table 5). Numbers of repeat PCI in the CHM plus RWM group was lower than that of the RWM alone group for $F u$ fang dan shen pill ( $\mathrm{RR}=0.14,95 \% \mathrm{CI}=0.03$ to 0.58 ) and Tong xin luo $(\mathrm{RR}=0.16,95 \% \mathrm{CI}=0.04$ to 0.68$)$. The remaining CHMs showed no statistically significant difference between the CHM plus RWM group and RWM alone group. But the overall result of their meta-analyses showed an obvious statistical difference between CHMs plus RWM versus RWM groups $(\mathrm{RR}=0.37,95 \% \mathrm{CI}=0.23$ to 0.59$)$.

Coronary Artery Bypass Graft. Coronary artery bypass graft (CABG) after index PCI was reported as a measure in 9 studies on 7 CHMs with 1141 patients (Table 5). CHM plus RWM was found to markedly reduce the risk of CABG over the same $\mathrm{RWM}$ alone $(\mathrm{RR}=0.29,95 \% \mathrm{CI}=0.09$ to 0.96$)$. But compared to RWM plus placebo, CHM Xiong shao capsule plus RWM could not reduce risk of CABG $(\mathrm{RR}=0.20,95 \% \mathrm{CI}$ $=0.02$ to 1.68 ).

3.4.6. Effect on Angina. Effect on angina was reported as a measure in 6 studies with 522 patients (Table 6). Two studies reported a followup period of 1 month, 2 studies reported a followup of 3 months, and 2 studies reported a followup of 6 months after index PCI. Angina improvement 
TABLE 4: Risk ratios of Chinese herbal medicines administered to prevent recurrent angina after PCI.

\begin{tabular}{|c|c|c|c|c|}
\hline Chinese herbal medicine & Number of studies & Number of patients & Risk ratio (RR) & $95 \% \mathrm{CI}$ of $\mathrm{RR}$ \\
\hline Bu xin yin plus RWM versus $\mathrm{RWM}$ & $1[76]$ & 44 & 0.60 & $0.33 \sim 1.10$ \\
\hline Dan hong tong mai capsule plus RWM versus RWM & $1[91]$ & 138 & 0.24 & $0.07 \sim 0.82$ \\
\hline Dan shen plus RWM versus RWM & $5[44,50,57,85,86]$ & 390 & 0.24 & $0.15 \sim 0.41$ \\
\hline Guan tong formula plus RWM versus RWM & $1[54]$ & 120 & 0.27 & $0.08 \sim 0.93$ \\
\hline Jia wei xue fu zhu yu particle plus RWM versus RWM & $1[51]$ & 60 & 0.45 & $0.18 \sim 1.15$ \\
\hline Jiang lian he ji plus RWM versus RWM & $1[63]$ & 69 & 0.71 & $0.30 \sim 1.70$ \\
\hline Lei gong teng plus RWM versus RWM & $1[64]$ & 75 & 0.39 & $0.17 \sim 0.94$ \\
\hline Liang xue shen ji formula plus RWM versus RWM & $1[46]$ & 97 & 0.52 & $0.16 \sim 1.65$ \\
\hline Shu xue tong injection plus RWM versus RWM & $1[56]$ & 43 & 0.37 & $0.11 \sim 1.25$ \\
\hline Shu xin yin plus RWM versus RWM & $1[75]$ & 44 & 0.60 & $0.33 \sim 1.10$ \\
\hline Tong guan capsule plus RWM versus RWM & $1[70]$ & 50 & 0.67 & $0.22 \sim 2.01$ \\
\hline Tong mai yi xin plus RWM versus RWM & $2[54]$ & 272 & 0.41 & $0.26 \sim 0.65$ \\
\hline Tong xin luo plus RWM versus RWM & $3[79,82,90]$ & 344 & 0.27 & $0.13 \sim 0.54$ \\
\hline Wen tong jian plus RWM versus RWM & $1[87]$ & 467 & 0.30 & $0.17 \sim 0.52$ \\
\hline Wen yang huo xue formula plus RWM versus RWM & $1[47]$ & 120 & 0.53 & $0.38 \sim 0.75$ \\
\hline Xue fu zhu yu pin plus RWM versus RWM & $2[72,84]$ & 157 & 0.44 & $0.25 \sim 0.78$ \\
\hline Xue yu tong he ji plus RWM versus RWM & $1[69]$ & 36 & 0.67 & $0.23 \sim 1.97$ \\
\hline Xue zhi kang plus RWM versus RWM & $1[48]$ & 60 & 0.38 & $0.16 \sim 0.94$ \\
\hline Xiong shao capsule plus RWM versus RWM & $2[40,80]$ & 173 & 0.48 & $0.31 \sim 0.75$ \\
\hline Yi qi hua yu formula plus RWM versus RWM & $1[41]$ & 60 & 0.44 & $0.15 \sim 1.29$ \\
\hline Yi xin capsule plus RWM versus RWM & $1[77]$ & 94 & 0.46 & $0.20 \sim 1.05$ \\
\hline Self-prepared guan tong decoction plus RWM versus RWM & $1[83]$ & 40 & 0.33 & $0.11 \sim 1.05$ \\
\hline Overall (CHM plus RWM versus $R W M)$ & 31 & 2953 & 0.40 & $0.35 \sim 0.47$ \\
\hline Xiong shao capsule plus RWM versus RWM plus placebo & $2[67,81]$ & 422 & 0.25 & $0.17 \sim 0.37$ \\
\hline CHM plus RWM versus RWM plus placebo & 2 & 422 & 0.25 & $0.17 \sim 0.37$ \\
\hline
\end{tabular}

$\mathrm{CHM}=$ Chinese herbal medicine; $\mathrm{CI}=$ confidence interval; $\mathrm{RWM}=$ routine Western medicine.

in these studies was defined as "significant improvement," "improvement," and "no improvement" based on Chinese herbal medicine clinical research guidelines [92]. To permit overall analysis, we converted these outcomes into dichotomous data. We grouped together "significant improvement" and "improvement" as "effective," and "no improvement" as "ineffective." There was no statistically significant difference between CHM plus RWM versus RWM groups for every CHM except for Shen mai gua lou shi xiao powder $(\mathrm{RR}=$ $1.55,95 \% \mathrm{CI}=1.11$ to 2.17$)$. Furthermore, the results were unsuitable for meta-analysis pooling due to heterogeneity $\left(I^{2}\right.$ $=60 \%)$.

3.4.7. Angiographic Measurements. Follow-up angiography was done on diffuse ISR 6 months after index PCI in 10 studies on 8 CHMs with 811 patients. Baseline information shows that the mean minimal luminal diameter (MLD) before and immediately after index PCI and gain in luminal diameter following stent placement were comparable between the comparison groups.

Minimum Lumen Diameter (MLD). Minimum lumen diameter is defined as smallest diameter of the lesion area being treated [93]. MLD was measured in 7 CHM studies (Table 7). The CHM plus RWM groups showed significant MLD improvement over the same RWM alone or plus placebo for
Fu fang dan shen capsule (MD (mean difference) $=0.05 \mathrm{~mm}$, $95 \% \mathrm{CI}=0.04$ to $0.26 \mathrm{~mm})$, Guan tong formula $(\mathrm{MD}=$ $0.21 \mathrm{~mm}, 95 \% \mathrm{CI}=0.06$ to $0.36 \mathrm{~mm}$ ) and Xiong shao capsule (placebo control; $\mathrm{MD}=0.49 \mathrm{~mm}, 95 \% \mathrm{CI}=0.12$ to $0.86 \mathrm{~mm}$ ). No significant difference was evident in the studies on Lei gong teng, Tong xin fang, Si ni tang. But in pooling the results of those trials, there was a statistically significant difference $(\mathrm{MD}=0.15 \mathrm{~mm}, 95 \% \mathrm{CI}=0.05$ to $0.24 \mathrm{~mm})$, with patients receiving CHMs more likely to have increased MLD than those in the control group.

Late Loss of Lumen (LLL). Late loss of lumen is defined as the decreased amount in lumen diameter after PCI, which is calculated by subtracting MLD at followup from MLD immediately post-procedure [93]. LLL was measured in 6 studies on 5 CHMs after 6 months' followup (Table 7). Five studies on 4 CHMs (Guan tong formula, Tong xin luo, Shu xue tong injection, and Lei gong teng) reported significantly better results for reducing late loss of lumen in the treatment group over the control group. There was no significant difference in the study on Fu fang dan shen capsule. The pooled results showed significant difference $(\mathrm{MD}=-0.24 \mathrm{~mm}, 95 \% \mathrm{CI}=$ -0.34 to $-0.15 \mathrm{~mm})$.

Net Gain in Lumen Diameter (NG). Net gain in lumen diameter is defined as the net increase in MLD after PCI [93]. 
TABLE 5: Risk ratios of Chinese herbal medicines administered to prevent MACE after PCI.

\begin{tabular}{|c|c|c|c|c|}
\hline Chinese herbal medicine & Number of studies & Number of patients & Risk ratio (RR) & $95 \% \mathrm{CI}$ of RR \\
\hline \multicolumn{5}{|l|}{ Acute myocardial infarction } \\
\hline $\mathrm{Bu}$ xin yin plus RWM versus $\mathrm{RWM}$ & $1[76]$ & 44 & 0.24 & $0.01 \sim 4.69$ \\
\hline Dan hong tong mai capsule plus RWM versus RWM & $1[91]$ & 138 & 0.14 & $0.01 \sim 2.64$ \\
\hline Dan shen plus RWM versus RWM & $2[44,85]$ & 168 & 0.14 & $0.02 \sim 1.11$ \\
\hline Fu fang dan shen pill plus RWM versus RWM & $2[50,57]$ & 156 & 0.32 & $0.03 \sim 2.99$ \\
\hline Ku lie zi injection plus RWM versus RWM & $1[78]$ & 40 & 0.33 & $0.01 \sim 7.72$ \\
\hline Shu xin yin plus RWM versus RWM & $1[75]$ & 44 & 0.24 & $0.01 \sim 4.69$ \\
\hline Tong xin luo plus RWM versus RWM & $2[79,90]$ & 268 & 0.25 & $0.03 \sim 2.22$ \\
\hline Xiong shao capsule plus RWM versus RWM & $2[40,80]$ & 173 & 0.14 & $0.02 \sim 1.06$ \\
\hline Yi xin capsule plus RWM versus RWM & $1[77]$ & 94 & 0.65 & $0.06 \sim 6.88$ \\
\hline Overall (CHMs plus RWM versus RWM) & 13 & 1125 & 0.22 & $0.10 \sim 0.49$ \\
\hline Xiong shao capsule plus RWM versus RWM plus placebo & $2[73]$ & 426 & 0.59 & $0.08 \sim 4.41$ \\
\hline Overall (CHMs plus RWM versus RWM plus placebo) & 2 & 426 & 0.59 & $0.08 \sim 4.41$ \\
\hline \multicolumn{5}{|l|}{ Revascularization } \\
\hline Shu xin yin plus RWM versus RWM & $1[75]$ & 44 & 0.69 & $0.23 \sim 2.01$ \\
\hline Bu xin yin plus RWM versus RWM & $1[76]$ & 44 & 0.69 & $0.23 \sim 2.01$ \\
\hline Yi xin capsule plus RWM versus RWM & $1[77]$ & 94 & 0.43 & $0.02 \sim 10.26$ \\
\hline Xiong shao capsule plus RWM versus RWM & $1[40]$ & 108 & 0.62 & $0.30 \sim 1.30$ \\
\hline Overall (CHMs plus RWM versus RWM) & 4 & 290 & 0.64 & $0.38 \sim 1.08$ \\
\hline Xiong shao capsule plus RWM versus RWM plus placebo & $2[67,81]$ & 426 & 0.48 & $0.30 \sim 0.78$ \\
\hline Overall (CHMs plus RWM versus RWM plus placebo) & 2 & 426 & 0.48 & $0.30 \sim 0.78$ \\
\hline \multicolumn{5}{|l|}{ Repeat PCI } \\
\hline Dan hong tong mai capsule plus RWM versus RWM & $1[91]$ & 138 & 0.39 & $0.08 \sim 1.94$ \\
\hline Dan shen plus RWM versus RWM & $1[52]$ & 80 & 1.0 & $0.21 \sim 4.66$ \\
\hline Fu fang dan shen pill plus RWM versus RWM & $2[50,57]$ & 156 & 0.14 & $0.03 \sim 0.58$ \\
\hline Ku lie zi injection plus RWM versus RWM & $1[78]$ & 40 & 0.33 & $0.01 \sim 7.72$ \\
\hline Lei gong teng plus RWM versus RWM & $1[43]$ & 80 & 0.56 & $0.20 \sim 1.51$ \\
\hline Liang xue shen ji formula plus RWM versus RWM & $1[46]$ & 97 & 0.54 & $0.14 \sim 2.14$ \\
\hline Si ni tang plus RWM versus RWM & $1[59]$ & 80 & 0.56 & $0.20 \sim 1.51$ \\
\hline Tong xin luo plus RWM versus RWM & $1[90]$ & 136 & 0.16 & $0.04 \sim 0.68$ \\
\hline Overall (CHMs plus RWM versus RWM) & 9 & 807 & 0.37 & $0.23 \sim 0.59$ \\
\hline \multicolumn{5}{|l|}{$C A B G$} \\
\hline Dan hong tong mai capsule plus RWM versus RWM & $1[91]$ & 138 & 0.14 & $0.01 \sim 2.64$ \\
\hline Dan shen plus RWM versus RWM & $1[52]$ & 80 & Not & estimable \\
\hline Fu fang dan shen pill plus RWM versus RWM & $2[50,57]$ & 156 & 0.32 & $0.03 \sim 2.99$ \\
\hline Liang xue sheng ji formula plus RWM versus RWM & $1[46]$ & 97 & 0.30 & $0.01 \sim 7.22$ \\
\hline Tong xin luo plus RWM versus RWM & $1[90]$ & 136 & 0.19 & $0.01 \sim 3.86$ \\
\hline Xiong shao capsule plus RWM versus RWM & $1[40]$ & 108 & 1.04 & $0.07 \sim 16.2$ \\
\hline Overall (CHMs plus RWM versus RWM) & 7 & 715 & 0.29 & $0.09 \sim 0.96$ \\
\hline Xiong shao capsule plus RWM versus RWM plus placebo & $2[67,81]$ & 426 & 0.20 & $0.02 \sim 1.68$ \\
\hline Overall (CHMs plus RWM versus RWM plus placebo) & 2 & 426 & 0.20 & $0.02 \sim 1.68$ \\
\hline
\end{tabular}

$\mathrm{CABG}=$ coronary artery bypass graft; $\mathrm{CHM}=$ Chinese herbal medicine; $\mathrm{CI}=$ confidence interval; $\mathrm{PCI}=$ percutaneous coronary intervention; $\mathrm{RWM}=$ routine Western medicine; MACE = major adverse cardiac event.

NG after 6 months' followup was measured in 4 studies on 4 CHMs (Table 7). Significant difference was found between the treatment group plus RWM and the control group with the same RWM alone for Guan tong formula (MD $=0.21 \mathrm{~mm}$, $95 \% \mathrm{CI}=0.08$ to $0.34 \mathrm{~mm})$ and Shu xue tong injection (MD $=0.25 \mathrm{~mm}, 95 \% \mathrm{CI}=0.09$ to $0.41 \mathrm{~mm})$. The pooled results of meta-analyses, suggested that CHMs plus RWM increased net gain of lumen compared to using RWM alone (MD $=0.2 \mathrm{~mm}, 95 \% \mathrm{CI}=0.11$ to $0.29 \mathrm{~mm})$. These results are displayed in Table 7.

Lesion Area Net Gain (LANG) . Lesion area net gain is defined as the net increase in lumen area before PCI and at followup after PCI $[53,55,64,82,86]$. Five studies on 
TABLE 6: Risk ratios of Chinese herbal medicines administered to prevent angina after PCI.

\begin{tabular}{|c|c|c|c|c|}
\hline Chinese herbal medicine & Number of studies & Number of patients & Risk ratio (RR) & $95 \% \mathrm{CI}$ of RR \\
\hline \multicolumn{5}{|l|}{ Followup 1 month } \\
\hline Shan shen tong mai he ji plus RWM versus RWM & $1[74]$ & 102 & 1.18 & $0.95 \sim 1.45$ \\
\hline Shen mai gua lou shi xiao power plus RWM versus RWM & $1[88]$ & 63 & 1.55 & $1.11 \sim 2.17$ \\
\hline Overall (CHMs plus RWM versus RWM)* & 2 & 165 & 1.31 & $1.00 \sim 1.72$ \\
\hline \multicolumn{5}{|l|}{ Followup 3 months } \\
\hline Tong guan capsule plus RWM versus RWM & $1[58]$ & 52 & 1.26 & $0.98 \sim 1.64$ \\
\hline Yi qu huo xue formula plus RWM versus RWM & $1[73]$ & 101 & 1.07 & $0.98 \sim 1.16$ \\
\hline Overall (CHMs plus RWM versus $R W M)^{*}$ & 2 & 153 & 1.13 & $0.93 \sim 1.36$ \\
\hline \multicolumn{5}{|l|}{ Followup 6 months } \\
\hline Xue fu zhu yu pill plus RWM versus RWM & $1[84]$ & 84 & 1.02 & $0.57 \sim 1.84$ \\
\hline Wen yang huo xue formula plus RWM versus RWM & $1[47]$ & 120 & 1.05 & $0.99 \sim 1.12$ \\
\hline Overall (CHMs plus RWM versus RWM) & 2 & 204 & 1.05 & $0.99 \sim 1.12$ \\
\hline Total overall (CHMs plus RWM versus RWM)* & 6 & 522 & 1.13 & $1.02 \sim 1.26$ \\
\hline
\end{tabular}

${ }^{*}$ Random effect model

$\mathrm{CHM}=$ Chinese herbal medicine; $\mathrm{CI}=$ confidence interval; $\mathrm{PCI}=$ percutaneous coronary intervention; $\mathrm{RWM}=$ routine Western medicine.

TABLE 7: Effect of Chinese herbal medicines on angiographic characteristics after PCI.

\begin{tabular}{|c|c|c|c|c|}
\hline Chinese herbal medicine & Number of studies & Number of patients & Mean difference & $95 \%$ CI of mean differ \\
\hline \multicolumn{5}{|l|}{ Minimum lumen diameter ( $\mathrm{mm}$ ) } \\
\hline Fu fang dan shen plus RWM versus RWM & $1[86]$ & 47 & 0.05 & $0.04 \sim 0.26$ \\
\hline Guan tong formula plus RWM versus RWM & $1[54]$ & 120 & 0.21 & $0.06 \sim 0.36$ \\
\hline Lei gong teng plus RWM versus RWM & $2[43,64]$ & 119 & 0.11 & $-0.13 \sim 0.36$ \\
\hline Si ni tang plus RWM versus RWM & $1[59]$ & 63 & 0.26 & $-0.03 \sim 0.55$ \\
\hline Tong xin fang plus RWM versus RWM & $1[49]$ & 61 & -0.10 & $-0.44 \sim 0.24$ \\
\hline Overall (CHMs plus RWM versus RWM) & 6 & 410 & 0.15 & $0.05 \sim 0.24$ \\
\hline Xiong shao capsule plus RWM versus RWM plus placebo & $1[67]$ & 97 & 0.49 & $0.12 \sim 0.86$ \\
\hline CHMs plus RWM versus RWM plus placebo & 1 & 97 & 0.49 & $0.12 \sim 0.86$ \\
\hline \multicolumn{5}{|l|}{ Late loss of lumen ( $\mathrm{mm})$} \\
\hline Fu fang dan shen plus RWM versus RWM & $1[86]$ & 47 & 0.00 & $-0.22 \sim 0.22$ \\
\hline Guan tong formula plus RWM versus RWM & $1[54]$ & 120 & -0.16 & $-0.28 \sim-0.04$ \\
\hline Lei gong ten plus RWM versus RWM & $1[64]$ & 53 & -0.30 & $-0.48 \sim-0.12$ \\
\hline Shu xue tong injection plus RWM versus RWM & $1[56]$ & 64 & -0.29 & $-0.44 \sim-0.14$ \\
\hline Tong xin luo plus RWM versus RWM & $2[55,82]$ & 148 & -0.33 & $-0.46 \sim-0.21$ \\
\hline Overall (CHMs plus RWM versus RWM)* & 6 & 432 & -0.24 & $-0.34 \sim-0.15$ \\
\hline \multicolumn{5}{|l|}{ Net gain in lumen diameter ( $\mathrm{mm}$ ) } \\
\hline Fu fang dan shen plus RWM versus RWM & $1[86]$ & 47 & 0.04 & $-0.18 \sim 0.26$ \\
\hline Guan tong formula plus RWM versus RWM & $1[54]$ & 120 & 0.21 & $0.08 \sim 0.34$ \\
\hline Lei gong tang plus RWM versus RWM & $1[64]$ & 53 & 0.28 & $-0.03 \sim 0.59$ \\
\hline Shu xue tong injection plus RWM versus RWM & $1[56]$ & 64 & 0.25 & $0.09 \sim 0.41$ \\
\hline Overall (CHMs plus RWM versus RWM) & 4 & 284 & 0.20 & $0.11 \sim 0.29$ \\
\hline \multicolumn{5}{|l|}{ Lesion area net gain $\left(\mathrm{mm}^{2}\right)$} \\
\hline Fu fang dan shen plus RWM versus RWM & $1[86]$ & 47 & 0.06 & $-0.54 \sim 0.66$ \\
\hline Guan tong formula plus RWM versus RWM & $1[54]$ & 120 & 0.66 & $0.25 \sim 1.07$ \\
\hline Lei gong teng plus RWM versus RWM & $1[64]$ & 53 & 0.66 & $0.41 \sim 0.91$ \\
\hline Tong xin luo plus RWM versus RWM & $2[55,82]$ & 148 & 0.99 & $0.64 \sim 1.33$ \\
\hline Overall (CHMs plus RWM versus RWM) & 5 & 368 & 0.69 & $0.52 \sim 0.87$ \\
\hline
\end{tabular}

${ }^{*}$ Random effect model

$\mathrm{CHM}=$ Chinese herbal medicine; $\mathrm{CI}=$ confidence interval; $\mathrm{PCI}=$ percutaneous coronary intervention; $\mathrm{RWM}=$ routine Western medicine. 
4 CHMs measured LANG at 6 months' followup after PCI (Table 7).The CHMs Guan tong formula, Tong xin luo, and Lei gong teng demonstrated a significant improvement in LANG. The same result was not found with the fourth CHM, $F u$ fang dan shen capsule. The pooled results of meta-analysis were statistically significant $\left(\mathrm{MD}=0.69 \mathrm{~mm}^{2}, 95 \% \mathrm{CI}=0.52\right.$ to $0.87 \mathrm{~mm}^{2}$ ).

3.4.8. Quality of Life. Two studies [45, 71] with randomized, double-blind and placebo-control design reported quality of life 1 month after PCI using the Chinese versions of the Seattle Angina Questionnaire (SAQ) and Short-Form 36 (SF36) Health Survey. The SAQ is a 19-item self-administered questionnaire that assesses how patients with $\mathrm{CHD}$ are fairing in five dimensions: physical limitation (PL), angina stability (AS), angina frequency (AF), treatment satisfaction (TS), and disease perception (DP). The results of comparing Xue fu zhu yu capsule plus RWM with the same RWM plus placebo suggested that receiving CHM had a positive effect on AS $(\mathrm{MD}=12.13,95 \% \mathrm{CI}=4.61$ to 19.65$)$ and $\mathrm{TS}(\mathrm{MD}=$ $12.13,95 \% \mathrm{CI}=6.19$ to 17.87$)$. However, in the study with Tong guan capsule, the results were contrary to that of Xue fu zhu yu capsule: AS ( $\mathrm{MD}=-36.2,95 \% \mathrm{CI}=-48.97$ to $-23.43)$, PL ( $\mathrm{MD}=-11.32,95 \% \mathrm{CI}=-19.38$ to -3.26$)$, AF $(\mathrm{MD}=-35.68,95 \% \mathrm{CI}=-50.16$ to -20.6$)$, TS $(\mathrm{MD}$ $=-25.03,95 \% \mathrm{CI}=-33.93$ to -16.13$)$, and $\mathrm{DP}(\mathrm{MD}=$ $-9.79,95 \% \mathrm{CI}=-35.95$ to -16.38$)$. Pooled results were not available because of heterogeneity and the $I^{2}(\%)$ ranging from $88 \%$ to $98 \%$.

SF-36 is a survey of patient health comprised of eight multiple-item scales measuring these dimensions: physical function (PF), role-physical (RP), bodily pain (BP), general health $(\mathrm{GH})$, vitality (VT), social function (SF), roleemotional (RE), and mental health $(\mathrm{MH})$. There is also a single-item measure that assesses health transition (HT). Patients receiving Tong guan capsule had low scores on all dimensions except for RP when compared to placebo control. Patients administered Xue fu zhu yu capsule scored high only on the RE dimension when compared to placebo control. We did not find any statistically significant difference between the treatment and placebo groups in the remaining dimensions. As with the SAQ, pooled results were not available because of heterogeneity with $I^{2}(\%)$ ranging from $85 \%$ to $98 \%$.

\section{Discussion}

In this systematic review, 52 studies accounting for 4905 CAD patients who underwent PCI were identified. Definitive randomization was found in 19 studies and 33 studies were found to be lacking definitive randomization. For the latter, we attempted to contact authors by telephone or e-mail for further information. But most replies were unsatisfactory and did not resolve our questions; other authors did not reply. Therefore, as a whole, the included studies were of low quality. Of all studies, only four were designed to compare CHMs plus RWM versus the same RWM plus placebo [45, $67,71,81]$. The remaining studies were designed to compare CHMs plus RWM versus the same RWM alone.
In the primary outcomes, 40 studies with 3805 patients assessed in-stent restenosis after PCI. Twenty of these studies involving $10 \mathrm{CHMs}$ showed clear evidence of decrease in restenosis rate. Furthermore, 13 of these studies were of low or moderate risk of bias over a minimum 6 months' followup (Xiong shao capsule [67, 80, 81], Dan shen [44, 52, 85], Guan tong formula [54], Bu xin yin [76], Jiang lian he ji [63] Shu xin yin [75], Tong guan capsule [70], self-prepared guan tong decoction [83], and Xue yu tong he ji [69],). Therefore, a moderate definitive conclusion can be drawn that CHMs are beneficial for preventing coronary restenosis after PCI. In particular, the CHM Xiong shao capsule, which was studied in 4 trials with 613 patients showed strong evidence with low risk of bias in preventing restenosis $[40,67,80,81]$. Other CHMs including Dan shen $[44,50,52,85,86]$ and Tong xin luo capsule $[55,79,82,90]$ showed the same significant result. But a definitive conclusion cannot be drawn because of limited numbers of patients in these trials as well as their low methodological quality.

We were unable to conclude whether CHMs decrease cardiac mortality during 6 months' followup after PCI. Although the result of meta-analysis showed a statistically significant difference between comparison groups, we could not assess a similar result in the analysis of any single relevant trial. This may be due to the limited patient numbers in these studies.

Adverse events in the treatment groups were generally higher than in controls for $5 \mathrm{CHMs}$, but a statistically significant difference was found in only one study on the CHM Lei gong teng [43]. Adverse reactions in these studies were reported as mild. Therefore, we feel further investigation is needed to confirm these reports.

In this review we also examined secondary outcomes, measures of effect post-PCI. Recurrent angina was followed up for 6 months in 33 studies involving 22 CHMs. In 21 of these studies with $11 \mathrm{CHMs}$, patients in the treatment group had significantly lower incidence of recurrent angina than those in the control group. Dan shen (capsule or pill) in 5 studies $[44,50,57,85,86]$, Xiong shao capsule in 4 studies $[40,67,80,81]$, Tong xin luo capsule in 3 studies [79, 82, 90], and Tong mai yu xin concentrated pill in 2 studies $[60,62]$ were significantly better than the control at reducing recurrent angina after PCI. Minimum lumen diameter in 7 studies with $6 \mathrm{CHMs}$, late loss of lumen in 6 studies with $5 \mathrm{CHMs}$, net gain in lumen diameter in 4 studies with $4 \mathrm{CHMs}$, and lesion area net gain in 5 studies with $4 \mathrm{CHMs}$ were measured, with results of meta-analyses being statistically significant. Studies on the following CHMs revealed angiographic results in the treatment groups were significantly better than in the control group: Guan tong formula [54] for minimum lumen diameter, late loss of lumen, net gain in lumen diameter, and lesion area net gain; Tong xin luo capsule [82] for late loss of lumen and area net gain in lumen diameter; Shu xue tong [56] for late loss of lumen and area net gain in lumen diameter; Xiong shao capsule [67] for minimum lumen diameter; Lei gong teng [64] for late loss of lumenand lesion area net gain.

Our meta-analysis found possible benefit in Chinese herbal medicine compared to control in the rates of 
restenosis, cardiac mortality, recurrent angina, and in MLD, NG, LLL, and LANG. Specifically, the CHMs Xiong shao capsule and Dan shen appeared to markedly reduce rates of restenosis and recurrent angina, and the CHM Tong xin luo was found to significantly reduce restenosis, recurrent angina, LLL, and LANG. The baseline characteristics of most studies, such as age, gender, severity of CAD, degree of coronary stenosis before PCI, and stent type were not significantly different between the treatment and control groups. Nevertheless, concluding that CHMs have definitive preventive effects on restenosis after PCI would be premature because most of the studies were of low quality with shortcomings such as inadequate concealment, nonreporting of dropouts, and their incomplete outcomes data point to the possibility of bias. Additionally, clinical heterogeneity was apparent because different categories of CHMs were used.

The key limitations of our review were quality of the included studies. Ideally, RCTs should adhere to known research design standards. For example, the medication, dosage, and course should be identical in the control groups, and when including patients with different levels of illness, the trial should use stratified randomization. Our examination of these studies did not find enough details of these characteristics though most of the included studies did report comparable baselines between comparison groups. Details about randomization methodology were also lacking. In the 52 studies we reviewed, 19 trials reported randomization using a random number table or computer random number generator such as SAS software, and 10 trials mentioned using sealed, opaque envelope concealment without further explanation. In addition, in some studies post-PCI angiographic assessment for restenosis was not carried out in comparable patient numbers between the study groups. For example, the authors of one study reported 30 patients in the treatment group and 45 patients in the control group. Post-PCI angiography was done on only 19 patients with 24 lesion vessels in the treatment group and on 26 patients with 29 lesion vessels in the control group, with 8 vessels in the treatment group and 13 vessels in control group determined to have restenosis [64]. No explanation was given as to why angiographic assessment was not carried out on comparable patient numbers. Thus this type of incomplete outcome data can lead to selection bias.

Studies that involve therapeutic trials should also report adverse events regardless of whether or not they occurred. Reporting of adverse effects is very important for evaluating the safety of interventional measures even though there is no certainty that the adverse event is related to the interventional measure. Furthermore, adverse events can affect study dropout rates. In our review, only 22 of the 52 trials we investigated reported adverse events, rendering it difficult to systematically evaluate the safety of CHMs for restenosis.

Most of the 52 studies did not mention type of stent deployed, bare metal versus drug eluting. Therefore, the level of effectiveness of CHMs is unknown when different stent types are used. Future research on this topic will help elucidate this.
Another area that did not receive attention in the studies is the effect of diabetes on restenosis. Persons with diabetes who undergo PCI with stent placement have a high rate of restenosis $[94,95]$. Though diabetes was included in baseline patient characteristics in the treatment and control groups in most of the 52 studies of this review, none of the reports indicated the impact diabetes may or may not have had on restenosis. Furthermore, in China, CHMs are widely used in the treatment of diabetes [26]. Researchers may want to factor in these issues when designing future studies.

\section{Conclusion and Recommendations}

From this review, we conclude CHMs may have moderate efficacy in preventing restenosis following percutaneous coronary intervention with stent placement. This is despite the fact that our investigation revealed unclear methodological quality, clinical heterogeneity, and some possible bias in the identified studies. Among the CHMs, Xiong shao capsule appears to be somewhat effective in preventing restenosis because studies involving this CHM were of low bias and had sufficient patient numbers. The CHM Dan shen (capsule or pill) appears to have latent beneficial efficacy in preventing restenosis because there were relatively more studies and patient numbers for this CHM. Therefore, we recommend that Dan shen should be a priority for further research. We did not find evidence of a beneficial effect for administering CHMs to prevent major adverse cardiac effects due to restenosis after PCI.

Future trials on CHMs as therapy to prevent restenosis post-PCI need to adhere to established design standards to overcome the limitations presented in this review. In particular, they should ensure adequate concealment of allocation and blinding of primary outcomes assessors.

\section{Abbreviations and Acronyms}

AMI: Acute myocardial infarction

CABG: Coronary artery bypass graft

CAD: Coronary artery disease

CHM: Chinese herbal medicine

DES: Drug-eluting stent

ISR: In-stent restenosis

LANG: Lesion area net gain

LLL: $\quad$ Late loss of lumen

MI: Myocardial infarction

MLD: Minimum lumen diameter

NG: Net gain in lumen diameter

PCI: Percutaneous coronary intervention

RCT: Randomized controlled trials

RR: $\quad$ Risk ratio

RWM: Routine western medicine.

\section{Conflict of Interests}

The authors declare no conflict of interests. 


\section{Funding}

Funding was provided by the National Natural Science Foundation of China (Grant no. 81072726, National Natural Science Foundation of China, Beijing, China).

\section{References}

[1] K. S. Reddy, "Cardiovascular disease in non-western countries," New England Journal of Medicine, vol. 350, no. 24, pp. 2438-2440, 2004.

[2] I. Aboderin, A. Kalache, Y. Ben-Shlomo et al., Life Course Perspectives on Coronary Heart Disease, Stroke and Diabetes, World Health Organization, Geneva, Switzerland, 2002.

[3] T. A. Gaziano, "Reducing the growing burden of cardiovascular disease in the developing world," Health Affairs, vol. 26, no. 1, pp. 13-24, 2007.

[4] E. D. Grech, "ABC of interventional cardiology: percutaneous coronary intervention. II: the procedure," British Medical Journal, vol. 326, no. 7399, pp. 1137-1140, 2003.

[5] X. Ma, T. Wu, M. P. Robich et al., "Drug-eluting stents," International Journal of Clinical and Experimental Medicine, vol. 3, no. 3, pp. 192-201, 2010.

[6] H. C. Lowe, S. N. Oesterle, and L. M. Khachigian, "Coronary in-stent restenosis: current status and future strategies," Journal of the American College of Cardiology, vol. 39, no. 2, pp. 183-193, 2002.

[7] G. W. Stone, S. G. Ellis, D. A. Cox et al., "A polymerbased, paclitaxel-eluting stent in patients with coronary artery disease," New England Journal of Medicine, vol. 350, no. 3, pp. 221-231, 2004.

[8] S. Aziz, J. L. Morris, and R. A. Perry, "Late stent thrombosis associated with coronary aneurysm formation after sirolimuseluting stent implantation," Journal of Invasive Cardiology, vol. 19, no. 4, pp. E96-E98, 2007.

[9] B. Lagerqvist, S. K. James, U. Stenestrand, J. Lindbäck, T. Nilsson, and L. Wallentin, "Long-term outcomes with drugeluting stents versus bare-metal stents in Sweden," New England Journal of Medicine, vol. 356, no. 10, pp. 1009-1019, 2007.

[10] J. F. Lassen, "Long-term safety and efficacy of drug-eluting stents," The Lancet, vol. 377, no. 9773, pp. 1213-1214, 2011.

[11] H. L. Kim, K. W. Park, J. J. Kwak et al., "Stent-related cardiac events after non-cardiac surgery: drug-eluting stent vs. bare metal stent," International Journal of Cardiology, vol. 123, no. 3, pp. 353-354, 2008.

[12] E. Camenzind, "Treatment of in-stent restenosis-back to the future?” New England Journal of Medicine, vol. 355, no. 20, pp. 2149-2151, 2006.

[13] M. E. Pfisterer, B. Richartz, and S. Silber, "The BASKET-LATE study: basel stent cost-effectiveness trial-late thrombotic events trial," Herz, vol. 31, no. 3, p. 259, 2006.

[14] J. M. Ten Berg, H. W. Thijs Plokker, and F. W. A. Verheugt, "Antiplatelet and anticoagulant therapy in elective percutaneous coronary intervention," Current Controlled Trials in Cardiovascular Medicine, vol. 2, no. 3, pp. 129-140, 2001.

[15] C. L. Grines, R. O. Bonow, D. E. Casey et al., "Prevention of premature discontinuation of dual antiplatelet therapy in patients with coronary artery stents," Circulation, vol. 115, no. 6, pp. 813-818, 2007.

[16] Q.-J. Meng, J. Wang, and R. Wu, "Progress of traditional Chinese medicine intervention after Percutaneous coronary intervention," China Journal of Chinese Materia Medica, vol. 33, no. 8, pp. 962-965, 2008.

[17] D. Ren, Y. Wu, J. Huang, and K. Wang, "Clinical study of Danshen Shuizi capsule in preventing restenosis after stent placement," China Journal of Traditional Chinese Medicine and Pharmacy, vol. 25, pp. 1518-1520, 2010.

[18] W. H. Wang, H. Y. Zhao, and J. M. Lang, "Effect of Shenqi decoction on hs-CRP, sIL-2R and activation of T lymphocytes after percutaneous coronary intervention," Chinese Journal of Integrative Medicine on Cardio-/Cerebrovascular Disease, vol. 8, pp. 413-415, 2010.

[19] X. Li, Z. Chen, G. S. Ma et al., "Experimental study of preventive effect of tetramethylpyrazine-eluting stent on instent restenosis," Journal of Southeast University, vol. 27, pp. 102-105, 2008.

[20] G. H. Tang, X. L. Tang, and G. H. Jiang, "Effect of perlolyrine and its analogues on platelet aggregation and experimental thrombosis," Chinese Pharmacological Bulletin, vol. 17, no. 3, pp. 333-336, 2001.

[21] B. Wu, M. Liu, H. Liu et al., "Meta-analysis of traditional Chinese patent medicine for ischemic stroke," Stroke, vol. 38, no. 6, pp. 1973-1979, 2007.

[22] W. Gong and Z. Chen, "Advancement of studies on Traditional Chinese medicine for restenosis after percutaneous coronary intervention," Zhejiang Journal of Integrated Traditional and Western Medicine, vol. 13, pp. 328-331, 2003.

[23] W. F. Zhang and Y. Yang, "Traditional Chinese medicine treating and preventing restenosis after percutaneous coronary intervention," Liaoning Journal of Traditional Chinese Medicine, vol. 32, pp. 84-85, 2005.

[24] Y. Ren, K. J. Chen, and X. M. Ruan, "Systematic review of randomized controlled trials on preventing and treating restenosis after percutaneous coronary intervention with Chinese medicine," Chinese Journal of Integrated Traditional and Western Medicine, vol. 28, no. 7, pp. 597-601, 2008.

[25] Chinese Society of Cardiology of Chinese Medical Association, "Editorial Board of Chinese Journal of Cardiology. Chinese Guidelines for percutaneous coronary intervention (2009)," Chinese Journal of Cardiology, vol. 37, pp. 4-25, 2009.

[26] J. P. Liu, M. Zhang, W. Y. Wang, and S. Grimsgaard, "Chinese herbal medicines for type 2 diabetes mellitus," Cochrane Database of Systematic Reviews, no. 3, article CD003642, 2004.

[27] J. Higgins and S. Green, "Assessing risk of bias in included studies," in Cochrane Handbook for Systematic Reviews of Intervenions Volume Version 502: the Cochrane Collaboration, J. Higgins and S. Green, Eds., 2009.

[28] J. Lau, J. P. A. Ioannidis, and C. H. Schmid, "Quantitative synthesis in systematic reviews," Annals of Internal Medicine, vol. 127, no. 9, pp. 820-826, 1997.

[29] J. P. T. Higgins, S. G. Thompson, J. J. Deeks, and D. G. Altman, "Measuring inconsistency in meta-analyses," British Medical Journal, vol. 327, no. 7414, pp. 557-560, 2003.

[30] D. B. Petitti, "Statistical methods in meta-analysis," in MetaAnalysis, Decision Analysis, and Cost-Effectiveness Analysis: Methods for Quantitative Synthesis in Medicine, D. B. Petitti, Ed., pp. 90-114, Oxford University Press, New York, NY, USA, 1994.

[31] J. Higgins and S. Green, Cochrane Handbook for Systematic Reviews of Interventions Version 5.0.2, The Cochrane Collaboration, 2009.

[32] X. Y. Cue, Q. Lin, Y. B. Nong et al., "Clinical study of lianxue shengji recipe on incidence of post-percutaneous coronary intervention restenosis and adverse cardiovascular events," Modern Chinese Medicine, vol. 11, pp. 40-42, 2009. 
[33] Q. Wang, Z. G. Yi, Y. Q. Shu et al., "Effect of guantong formula on hs-CRP after intracoronary bare stenting," Journal of New Chinese Medicine, vol. 42, pp. 11-13, 2010.

[34] Z. Q. Qiao, M. Z. Zhang, H. Liu, K. L. Chen, and S. Li, "A randomized double-blinded placebo-controlled clinical study of Tongguan capsule improving cardiac functional parameter of patients after PCI," Chinese Archives of Traditional Chinese Medicine, vol. 21, pp. 882-883, 2003.

[35] H. Y. Wang, T. N. Fang, F. Wu, J. Zhao, Y. M. Tan, and X. Z. Zhang, "Study on the effects on hemorheology of CHD patients by Tonifying qi and activating blood after percataneous coronary intervention," Liaoning Journal of Traditional Chinese Medicine, vol. 36, pp. 397-398, 2009.

[36] X. Wang, Z. X. Lin, J. B. Ge, Y. He, L. Shen, and Z. J. Zhang, "The change of primary asthenia-secondary sthenia syndrome of coronary heart disease before and after stenting and therapical function of shuxin yin," Chinese Journal of Information, vol. 9, pp. 13-15, 2002.

[37] K. J. Chen, D. Z. Shi, and H. Xu, "Multi-central randomized double-blinded controlled trial of Xiong shao capsule preventing restenosis of patients with coronary heart disease after percutaneous coronary intervention," in Proceedings of the 6th National Congress on Blood Stasis Syndrome, pp. 23-28, Beijing, China, 2005.

[38] Y. He, X. Wang, Z. X. Lin, and J. B. Ge, "Effect of Shuxinyin combination therapies for restenosis after PCI," Journal of Traditional Chinese Medicine, vol. 43, pp. 701-703, 2002.

[39] J. C. Song, G. M. Hang, Z. R. Ding, C. H. Zheng, and J. T. Jie, "Effects of shuxuetong on acute coronary syndrome patients after interventional therapy," Shanxi Medical Journal, vol. 38, pp. 439-441, 2009.

[40] H. Xu, K. J. Chen, D. Z. Shi, and J. M. Mao, "Clinical study of xiongshao capsule in preventing restenosis after coronary interventional treatment," Chinese Journal of Integrated Traditional and Western Medicine, vol. 8, pp. 162-166, 2002.

[41] H. An, C. M. Mu, and X. S. Li, "Preventive effect of qisupplementing and stasis-resolving prescription on restenosis of 60 patients with coronary heart disease after percutaneous coronary intervention," Traditional Chinese Medicinal Research, vol. 22, pp. 26-27, 2009.

[42] Z. M. Bao, S. L. Wu, X. J. Shi et al., "The clinical observation on puerarin preventing restenosis after percutaneous coronary intervention in patients with acute coronary syndrone," Journal of Emergency in Traditional Chinese Medicne, vol. 14, pp. 613-614, 2005.

[43] G. H. Chen and K. Y. Song, "A sustained administration of tripterygium glycosides to reduce restenosis after coronary stenting," ACTA Medicinae Sinica, vol. 18, pp. 507-509, 2005.

[44] H. Chen, Z. A. Yi, S. Y. Yi, W. P. Hu, and F. Yi, "Efficacy of danshen injection after coronary stent implantation," Shanghai Medical and Pharmaceutical Journal, vol. 26, pp. 174-176, 2005.

[45] F. Y. Chu, J. Wang, X. W. Sun et al., "A randomized doubleblinded controlled trial of Xuefu Zhuyu Capsule on shortterm quality of life in unstable anginal patients with bloodstasis syndrome after percutaneous coronary intervention," Journal of Chinese Integrative Medicine, vol. 7, no. 8, pp. 729735, 2009.

[46] X. Y. Cui, Y. Wu, Y. B. Nong et al., "Effect of liangxue shengji recipe on incidence of post-percutaneous coronary intervention restenosis and adverse cardiovascular events," Chinese Journal of Integrated Traditional and Western Medicine, vol. 30, pp. 30-32, 2010.
[47] D. W. Dan, Clinical study on wenyang huoxue decoction to impact yang deficiency and blood stasis of post percutaneous coronary intervention patients, M.S. thesis, Guangzhou University of Chinese Medicine, Guangzhou, China, 2009.

[48] S. Y. Ding, P. Wan, L. X. Guan, X. Y. Yang, S. Y. He, and Y. Q. Cui, "Investigation of the effect of xuezhikang on the prevention for the restenosis after PCI with non-drug eluting stent in patients with coronary heart disease," China Practical Medicine, vol. 2, pp. 12-14, 2007.

[49] Q. M. Feng, Y. R. Dong, and Z. F. Yang, "Effect of tongxinfang on coronary collateral formation and stent-restenosis in patients with coronary artery disease after stenting," China Journal of Rehabilitation and Practice, vol. 12, pp. 152-153, 2006.

[50] G. S. Fu, "Clinical observation of compound Danshen drop pill preventing restenosis of patients with coronary heart disease after stenting operation (Part 1)," China Medical Herald, vol. 6, pp. 68-69, 2009.

[51] X. Gu, S. Z. Shang, J. L. Gao et al., "Jiawei xuefu zhuyu drop treating and preventing restenosis after stenting intervention," Chinese Journal of Integrative Medicine on Cardio/Cerebrovascular Disease, vol. 5, pp. 1042-1043, 2007.

[52] L. E. Guo, X. H. Chen, and R. S. Zhang, "Far prognostic clinical study on patients of medicinal bracket operation of coronary artery with salvia tablet," Journal of Zhejiang University of Traditional Chinese Medicine, vol. 33, pp. 327328,2009 .

[53] Y. J. Han, Clinical study on intervention of Xuesaitong soft capsule on coronary heart disease patient after PCI, M.S. thesis, Guangzhou University of Chinese Medicine, Guangzhou, China, 2008.

[54] J. S. He, Q. Wang, Z. G. Yi et al., "Effect of guantong formula on restenosis after intracoronary bare stenting," Journal of Traditional Chinese Medicine, vol. 51, pp. 423-425, 2010.

[55] Y. Kai, H. L. Liu, and Y. M. Zhao, "Clinical research of preventing post-PCI restenosis with tongxinluo capsule and complex dripping pill danshen," Chinese Journal of Practical Medicine, vol. 35, pp. 24-26, 2008.

[56] A. H. Li, K. Z. Gong, J. F. Yan, X. Sun, Y. Feng, and Z. Zhang, "Effect of shuxuetong in preventing restenosis after intracoronary stenting," Chinese Journal of Integrated Traditional and Western Medicine, vol. 24, no. 10, pp. 879-881, 2004.

[57] G. H. Li, G. S. Fu, and X. Q. Zhou, "Clinical observation of compound danshen drop pill preventing restenosis of patients with coronary heart disease after stenting operation (Part 2)," Modern Journal of Integrated Traditional Chinese and Western Medicine, vol. 19, pp. 920-921, 2010.

[58] J. Li, W. H. Zhang, M. Z. Zhang, and X. Z. Lin, "Clinical observation of Tongguan capsule treating restenosis of 26 patients with syndrome of deficiency of vital energy and blood stasis after PCI," New Journal of Traditional Chinese Medicine, vol. 37, pp. 33-35, 2005.

[59] L. T. Li and K. Y. Song, "Effect of sini tang on restenosis after cytoskeletal operation on coronary artery," Journal of Changchun University of Traditional Chinese Medicine, vol. 22, pp. 8-9, 2006.

[60] Q. H. Li, X. B. Li, Q. Y. Li et al., "Clinical study on tongmaiyixin concentrated pill to prevent restenosis after intracoronary stenting," China Medicine Herald, vol. 5, pp. 6$8,2008$.

[61] X. B. Li, "Observation of Xingmai futong decoction for prevention and treatment of restenosis of coronary artery 
cardle operation," Modern Medicine \& Health, vol. 20, p. 1858, 2004.

[62] X. B. Li, "Clinical observation and effect of TXA2, PGI2 on Tongmaiyuxin concentrated pill treating and preventing restenosis after PCI," Guangming Journal of Chinese Medicine, vol. 24, pp. 1629-1630, 2009.

[63] Y. Li and J. H. Niu, "The preventive effects of on re-stricture viscosity reduction mixture after percutaneous intracavitary plastic operation," Journal of Shandong Medical College, vol. 30, pp. 229-232, 2008.

[64] J. Liu, X. H. Fu, W. L. Wu et al., "Influence of tripterygium polyglycosides on restenosis after intracoronary stenting," Journal of Chinese Integrative Medicine, vol. 22, pp. 894-898, 2002.

[65] Y. J. Liu, G. Y. Sun, Z. Y. Liu, S. Z. Zhang, and S. L. Liang, "Clinical effects of tetramethyl pyrazine on preventing restenosis after percutaneous coronary intervention," Chinese Journal of Cardiovascular Medicine, vol. 9, pp. 90-92, 2004.

[66] S. B. Liu, J. S. Li, and Y. Zhang, "Clinical experience about Chinese herbal medicine with effects of replenishing qi and activating blood preventing on restenosis after percutaneous transluminal coronary angioplasty," Chinese Archives of Traditional Chinese Medicine, vol. 25, pp. 2556-2558, 2007.

[67] X. Y. Lu, D. Z. Shi, and H. Xu, "Clinical study on effect of xiongshao capsule on restenosis after percutaneous coronary intervention," Chinese Journal of Integrated Traditional and Western Medicine, vol. 26, no. 1, pp. 13-17, 2006.

[68] S. H. Ma, "Clinical observation of Guanmai zhaitong decoction for prevention and treatment of restenosis of coronary artery after cradle operation," Chinese Journal of Integrative Medicine on Cardio-/Cerebrovascular Disease, vol. 7, p. 1389, 2009.

[69] J. H. Niu, D. Li, S. Xi, and X. Y. Liu, "Clinical study on xueyutong mixture preventing coronary restenosis after plastic and cytoskeletal operation in coronary heart disease," Shandong Journal of Traditional Chinese Medicine, vol. 22, pp. 395-397, 2003.

[70] J. Y. Qi, M. Z. Zhang, J. Li, B. J. Chen, and K. L. Chen, "Effect of Tongguan capsule on restenosis and blood rheology after pecutaneous coronary intervention," Chinese Archives of Traditional Chinese Medicine, vol. 21, pp. 882-883, 2003.

[71] Z. Q. Qiao, M. Z. Zhang, X. W. Zhang, S. Li, and K. L. Chen, "A randomized double-blinded placebo-controlled clinical study of Tongguan capsule improving life quality of patients after PCI," Chinese Journal of Integrative Medicine on Cardio/Cerebrovascular Disease, vol. 4, pp. 4-5, 2006.

[72] D. Z. Shi, J. Li, X. C. Ma et al., "Clinical observation on prevention of restenosis in cases with coronary heart disease after percutaneous transluminal coronary angioplasty with concentrated Xuefu Zhuyu Pill," Journal of Traditional Chinese Medicine, vol. 38, pp. 27-29, 1997.

[73] H. Y. Wang, T. N. Fang, and F. Wu, "Effect of therapy of strengthening qi and activating blood on cardiac function in patients with coronary heart disease after percutaneous coronary intervention," Journal of New Chinese Medicine, vol. 41, pp. 39-41, 2009.

[74] Q. Wang, X. R. Xu, Z. Y. Wang et al., "Clinical observation of Shanshen tongmai composition treating the angina of patients after stenting intervention," Chinese Journal of Information on Traditional Chinese Medicine, vol. 13, pp. 68-69, 2006.

[75] X. Wang, Z. X. Lin, J. B. Ge et al., "Preliminary observation on application of shuxinyin after coronary artery stenting," Journal of Chinese Integrative Medicine, vol. 22, pp. 809-812, 2002.
[76] X. Wang, L. R. Yun, Z. X. Lin, and J. B. Ge, "Effect of buxinyin-chaifang on the inflammatory factors in patients after coronary artery stenting," Chinese Journal of Gerontology, vol. 23, pp. 144-146, 2003.

[77] X. T. Wang and Y. H. Gao, "Influence of yixin capsule on restenosis after intracoronary stenting," Chinese Journal of Cardiovascular Review, vol. 2, pp. 860-862, 2004.

[78] Z. H. Wang, J. Zhang, and Y. Xing, "Effect of Kudiezi injection on stent thrombosis and matrix metalloproteinase in patients with PCI," Journal of Jilin University Medicine Edition, vol. 36, no. 2, pp. 377-380, 2010.

[79] H. B. Xiao, D. D. Zhang, and J. Gu, "Effects of tongxinluo on C-reactive protein and clinical prognosis in patients after coronary stenting," Journal of Interventional Radiology, vol. 16, no. 8, pp. 520-522, 2007.

[80] H. Xu, D. Shi, and K. Chen, "Clinical effect of Xiongshao capsule on preventing restenosis post-PTCA or/and stenting," Chinese Journal of Integrated Traditional and Western Medicine, vol. 20, no. 7, pp. 494-497, 2000.

[81] K. J. Chen, D. Z. Shi, H. Xu et al., "XS0601 reduces the incidence of restenosis: a prospective study of 335 patients undergoing percutaneous coronary intervention in China. Chin Med J,2006;119:6-13. *Xu H, Lu XY, Chen KJ, et al. Study on correlation of blood-stasis syndrome and its accompanied syndromes with pathological changes showed in coronary angiography and restenosis after percutaneous coronary intervention," Chinese Journal of Integrated Traditional and Western Medicine, vol. 27, no. 8, pp. 13-2007, One trial reported in two publications each describing different outcome measures.

[82] F. M. Yao, L. Liu, and G. Y. Ge, "Clinical study of Tong xin lou capsule in preventing restenosis in patients with coronary heart disease," Chinese Journal of Difficult and Complicated Cases, vol. 5, pp. 191-192, 2006.

[83] Z. G. Yi, J. S. He, Q. Wang, L. Q. Shu, and L. Q. Yang, "Clinical efficacy of self-prepared guantong decotion for prevention PTCA and stenosis after coronary stenting," Journal of Changchun University of Traditional Chinese Medicine, vol. 8, pp. 33-35, 2005.

[84] B. Yu, K. Chen, and J. Mao, "Clinical study on effect of concentrated xuefu zhuyu pill on restenosis of 43 cases coronary heart disease after intracoronary stenting," Chinese Journal of Integrated Traditional and Western Medicine, vol. 18, no. 10, pp. 585-589, 1998.

[85] Z. A. Yu and S. Y. Yu, "Preliminary observation on oral danshen pian after coronary artery stenting," Shanghai Medical Journal, vol. 29, pp. 280-282, 2006.

[86] Z. H. Zheng, M. S. Wu, T. W. Liu, P. Li, L. G. Zhu, and D. J. $\mathrm{Zhu}$, "The effect of probucol and fu fang dan shen drops alone and incombination on restenosis after intracoronary stenting," Journal of Guangxi Medical University, vol. 21, pp. 809-814, 2004.

[87] Z. M. Zhang, Y. X. Gao, H. L. Liu, Y. Q. Yuan, and L. Yu, "Wentongjian in prevented coronary restenosis of percutaneous coronary intervention," Chinese Journal of Arteriosclerosis, vol. 14, pp. 610-612, 2006.

[88] W. M. Zhang, W. Zhang, and J. Zheng, "Clinical study of Shenmai gualou shixiao power treating recurrence angina after coronary in-stent," Journal of Traditional Chinese Medicine University of Hunan, vol. 27, pp. 49-51, 2007.

[89] H. Y. Zhao, W. H. Wang, J. M. Lang et al., "The effect mechanism of shenqi decoction on restenosis after implantation of 
stents in coronary artery disease," Chinese Journal of Integrative Medicine on Cardio-/Cerebrovascular Disease, vol. 7, pp. 382384, 2009.

[90] J. Zhou and J. T. Guo, "Clinical study of tongxinluo on preventing re-stenosis of stents in CHD," Heibei Medicine, vol. 13, pp. 1187-1190, 2007.

[91] L. Y. Zhu, L. Yan, and Z. W. Yang, "Clinical study of Danhong tongmai capsule preventing restenosis in stent and forming of late coronary thrombosis," Chinese Journal of Basic Medicine in Traditional Chinese Medicine, vol. 15, pp. 682-683, 2009.

[92] X. Y. Zheng, Guiding Principle of Clinical Research on New Drugs of Traditional Chinese Medicine, Chinese Medicine Science and Technology Press, Beijing, China, 2002.

[93] R. E. Kuntz and D. S. Baim, "Defining coronary restenosis: newer clinical and angiographic paradigms," Circulation, vol. 88, no. 3, pp. 1310-1323, 1993.

[94] J. Gilbert, J. Raboud, and B. Zinman, "Meta-Analysis of the effect of diabetes on restenosis rates among patients receiving coronary angioplasty stenting," Diabetes Care, vol. 27, no. 4, pp. 990-994, 2004.

[95] L. Martínez-Elbal, J. M. Ruiz-Nodar, J. Zueco et al., "Direct coronary stenting versus stenting with balloon pre-dilation: immediate and follow-up results of a multicentre, prospective, randomized study. The DISCO trial," European Heart Journal, vol. 23, no. 8, pp. 633-640, 2002. 


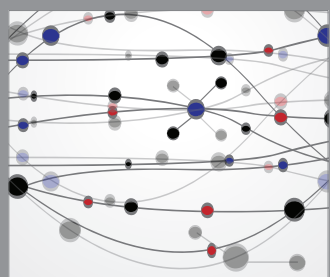

The Scientific World Journal
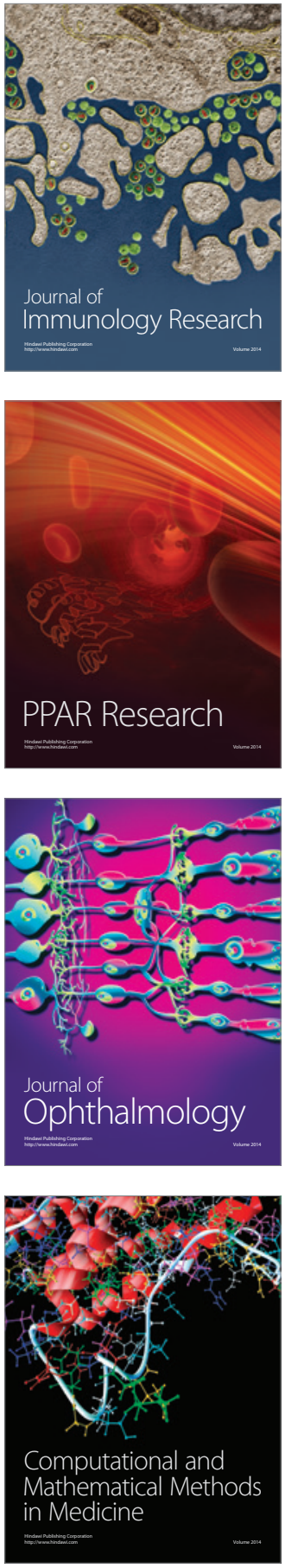

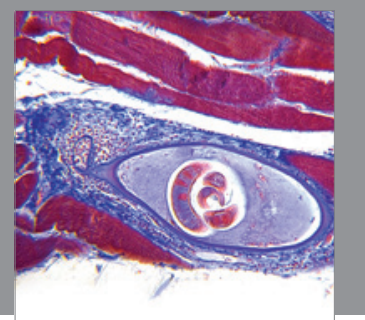

Gastroenterology

Research and Practice
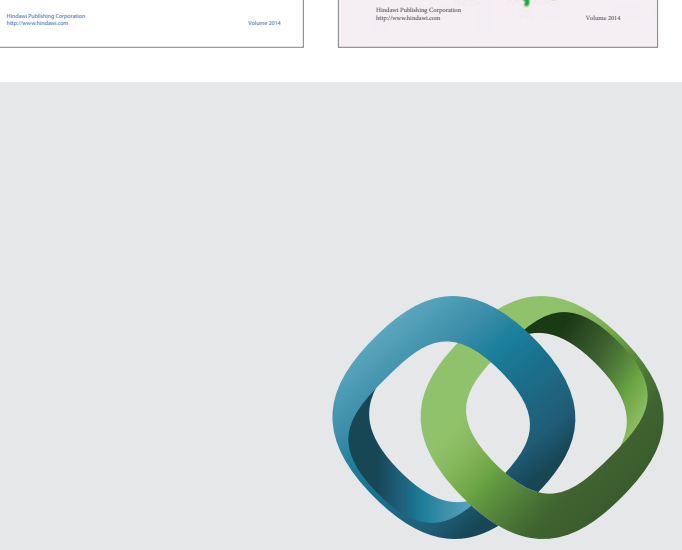

\section{Hindawi}

Submit your manuscripts at

http://www.hindawi.com
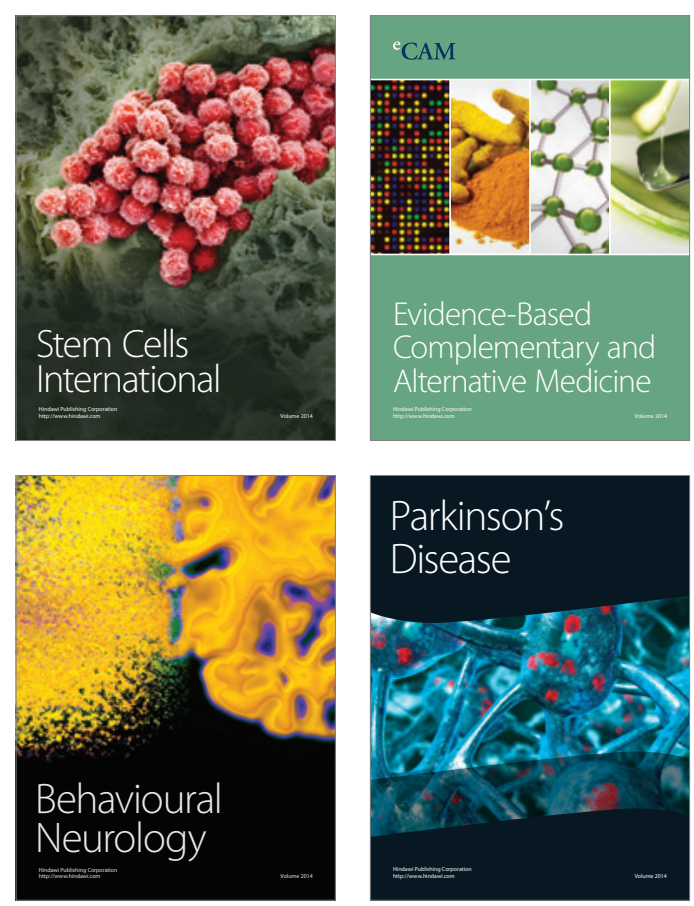

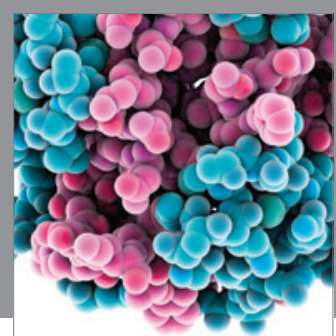

Journal of
Diabetes Research

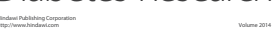

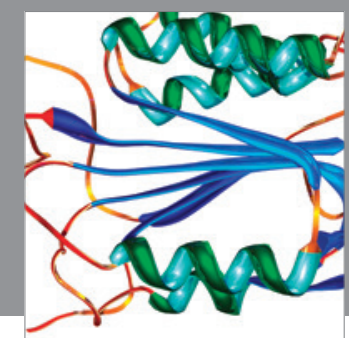

Disease Markers
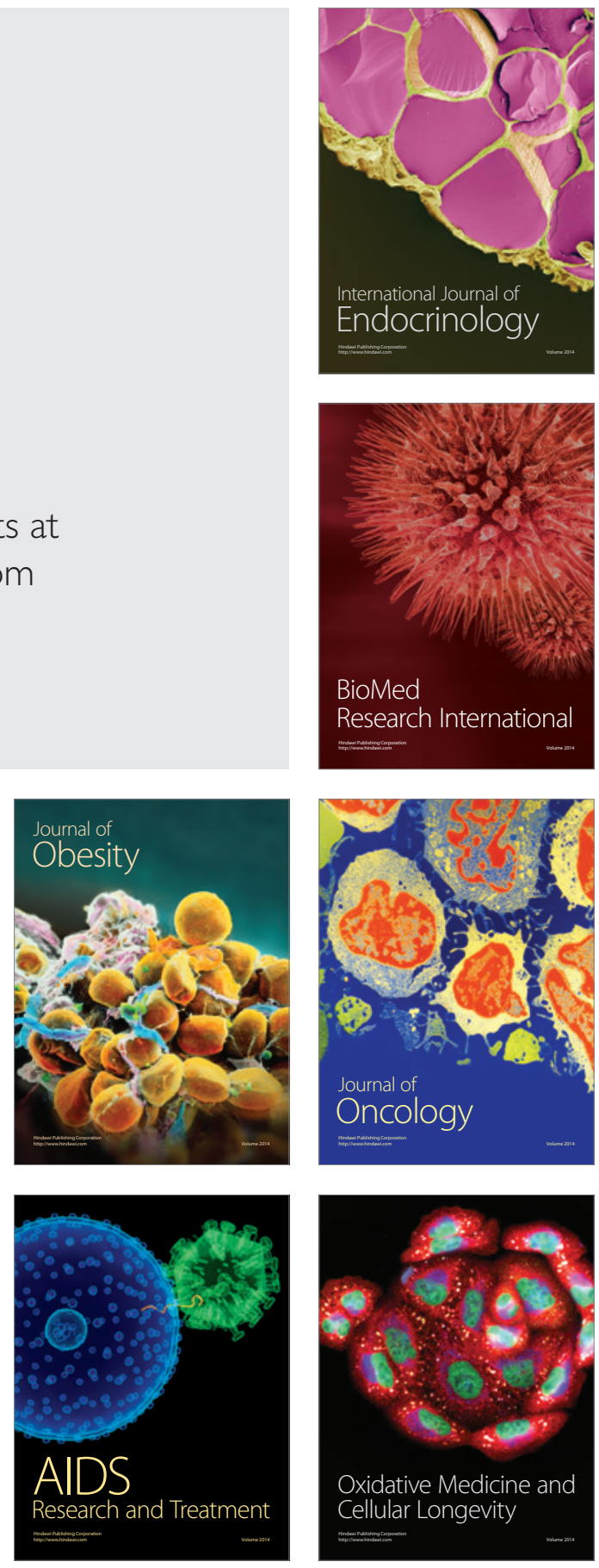